\title{
In welchen Betrieben verdienen Frauen mehr? Der Einfluss betrieblicher Statusrelationen auf die geschlechtsspezifische Lohnungleichheit ${ }^{* *}$
}

\section{Zusammenfassung}

Dieser Beitrag untersucht, inwiefern die geschlechtsspezifische Lohnungleichheit in Deutschland von statusbasierten Ansprüchen im Betrieb abhängig ist. Grundsätzlich sollte sich die geschlechtsspezifische Lohnungleichheit verringern, wenn sich der Status von Frauen im Betrieb relativ zu ihren männlichen Kollegen erhöht. Es werden zwei Indikatoren für diese betrieblichen Statusrelationen entlang der Geschlechtskategorie betrachtet: Frauenanteil im Management und Unterschiede zwischen den Geschlechtern beim Besitz von Hochschulabschlüssen. Mit der Verdienststrukturerhebung von 2010 und Multilevel Modellen kann gezeigt werden, dass sich der relative Lohn von Frauen mit steigendem Frauenanteil im Management und mit zunehmenden Vorteilen beim Besitz von Hochschulabschlüssen erhöht. Diese positiven Effekte auf den Lohn von Frauen variieren zudem entlang der Lohnverteilung: Bildungsvorteile von Frauen wirken sich stärker positiv auf hohe Löhne aus, während der Frauenanteil im Management eher unteren Lohngruppen hilft. Schließlich sind die Effekte stärker ausgeprägt in Betrieben ohne Tarifbindung.

Keywords: geschlechtsspezifische Lohnungleichheit; Theorie relationaler Ungleichheit; Statusrelationen; Tarifbindung; Betriebe

In which firms do female workers earn more? The influence of status relations in the firm on the gender wage gap

\begin{abstract}
Relational inequality theory suggests that status-based claims on organizational resources are a central mechanism leading to wage inequality at the workplace. Following this theory the paper examines the relationship between status-based claims in the firm and the gender wage gap in Germany. In general, it is expected that wage inequality between women and men decreases, when the status of women increases relative to male coworkers in the firm. I look at two indicators of status relations along the gender category: share of females in management and differences in the share of university degrees in the firm. Using the German Structure of Earnings Survey 2010 and employing multi-level regression models I find that the gender wage gap decreases with an increasing share of females in management
\end{abstract}

* Michael Schweiker. E-Mail: michael.schweiker@outlook.com

** Artikel eingegangen: 21.7.2016; revidierte Fassung nach doppelt-blindem Begutachtungsverfahren akzeptiert: 5.9.2017. 
and with growing differences between the sexes in the share of university degrees. In addition, these positive effects on the wage of female employees vary along the wage distribution: The effect of advantages in university degrees is stronger for higher wages, whereas the share of female managers predominantly helps low wage groups. Finally, the effects are stronger in firm with individual wage determination compared to firms that are covered by a collective agreement.

Keywords: gender wage gap; relational inequality theory; categorical status distinctions; coverage by collective wage agreements; work organizations

JEL-Deskriptoren: D310, D330, J310, J510

\section{Einleitung}

Nach wie vor verdienen Frauen in Deutschland weniger als ihre männlichen Kollegen. Die unbereinigte geschlechtsspezifische Lohnungleichheit (gender wage gap, GWG) gemessen am Bruttostundenlohn liegt seit Jahren bei über 20\%. ${ }^{1}$ Deutschland gehört damit im europäischen Vergleich zu den Ländern mit der größten Lohnungleichheit zwischen den Geschlechtern. Erklärungsversuche greifen zumeist auf das Humankapitalframework zurück: Die Lohnungleichheit ist demnach eine Folge unterschiedlicher Investitionsentscheidungen in Humankapital und sich akkumulierender Unterschiede im Lebenslauf. Daneben werden auch die Segregation in bestimmte (geringer entlohnende) Berufe, Branchen und Unternehmen und Diskriminierung als mögliche Ursachen diskutiert (vgl. Blau \& Kahn, 2016 für einen Überblick).

In den letzten Jahren wird aber verstärkt darauf hingewiesen, dass der Lohnbildungsprozess, wie ihn sich die Humankapitaltheorie vorstellt, eine zu grobe Vereinfachung darstellt (Lips, 2013). Insbesondere hat sich die Erkenntnis durchgesetzt, dass die Arbeitsorganisation ein zentraler Ort der Produktion sozialer Ungleichheit im Allgemeinen und geschlechtsspezifischer Lohnungleichheit im Besonderen ist (Acker, 1990; Baron \& Bielby, 1980; Baron, 1984; Tomaskovic-Devey, 2014). Mit der Theorie der relationalen Ungleichheit (Avent-Holt \& Tomaskovic-Devey, 2014; Nelson \& Bridges, 1999; Stainback, Tomaskovic-Devey \& Skaggs, 2010; Tomaskovic-Devey, 2014) wird hier auf ein theoretisches Modell zurückgegriffen, das explizit den Betrieb als Ort der Produktion von Lohnungleichheit konzeptualisiert, und dabei andere Lohnsetzungsmechanismen vorschlägt. Akteure im Betrieb erheben Anspruch auf organisationale Güter. Dabei stehen den Akteuren Ressourcen in Form von Statuskategorien (Managementposition, Bildungstitel) zur Verfügung, um ihre Ansprüche auf diese organisationalen Güter durchzusetzen. Daraus ergibt sich die zentrale Implikation des Modells: Die geschlechtsspezifische Lohnungleichheit nimmt in dem Maße ab, wie sich die Statusrelationen zwischen den Geschlechtern im Betrieb zugunsten von Frauen verschieben.

Der vorliegende Beitrag überprüft, inwiefern zwei betriebliche Statusrelationen, nämlich der Frauenanteil im Management und die Differenz beim Besitz von Hochschulabschlüssen, einen Zusammenhang mit der geschlechtsspezifischen Lohnungleichheit aufweisen und ob dieser entlang der Lohnverteilung und zwischen tarifgebundenen Betrieben und

1 Nach aktuellen Zahlen des Statistischen Bundesamts (2016) liegt der GWG bei 21\%. 
Betrieben mit individuellen Lohnverhandlungen variiert. Die Implikationen des relationalen Modells werden mit der Verdienststrukturerhebung von 2010 getestet. Dabei handelt es sich um einen administrativen, verknüpften Arbeitnehmer-Arbeitgeber-Datensatz, der sich aufgrund seiner unzensierten Einkommensangaben sehr für Verdienstanalysen eignet. Die Ergebnisse weisen auf die Bedeutung von betrieblichen Statusrelationen hin: Je höher der Frauenanteil im Management und je größer die Bildungsvorteile von Frauen relativ zu Männern im Betrieb, desto höher ist der relative Lohn von Frauen - insbesondere in Betrieben ohne Tarifbindung.

Der Beitrag ist wie folgt gegliedert: In Abschnitt 2 wird die Theorie der relationalen Ungleichheit vorgestellt. In Abschnitt 3 werden Hypothesen abgeleitet und der zugehörige Forschungsstand diskutiert. Abschnitt 4 enthält eine Beschreibung der verwendeten Daten und der Analysestrategie. In Abschnitt 5 werden die Ergebnisse präsentiert und Abschnitt 6 fasst sie zusammen.

\section{Die Theorie der relationalen Ungleichheit: Wie entsteht Lohnungleichheit im Betrieb?}

Wie ist zu erklären, dass der GWG unabhängig vom der Humankapitalausstattung zwischen verschiedenen Betrieben schwankt? Eine zentrale Antwort ist Diskriminierung. Hinter diesem Begriff versteckt sich allerdings eine Reihe von Ansätzen, von denen Beckers Diskriminierungsmodell einer der bekanntesten ist (Becker, 1971): Diskriminierende Arbeitgeber stellen weniger Frauen ein und zahlen ihnen geringere Löhne bzw. den männlichen Kollegen eine zusätzliche Kompensation dafür, dass sie mit Frauen arbeiten „müssen“. Sie verhalten sich also so, als verursachten Frauen Kosten. Die Implikationen des Modells wurden bereits häufig mit gemischten Resultaten getestet (z.B. Heinze, 2009; Ludsteck, 2014) was nicht zuletzt an einem großen Selektivitätsproblem und dem Problem der Messung von Diskriminierungsneigung liegt. Davon abgesehen berücksichtigt ein solches Modell auch nicht die vielfältigen und an den jeweiligen betrieblichen Kontext gebundenen Interaktionsprozesse, die hinter der Präferenz für Diskriminierung stehen, die im Zentrum der nun zu beschreibenden Theorie stehen.

\subsection{Claims-making}

Die zentrale, genuin soziologische Annahme der Theorie der relationalen Ungleichheit (relational inequality theory, RIT) ist der relationale Charakter von Ungleichheit (Tilly, 1998). Lohnungleichheit in Erwerbsorganisationen resultiert nicht aus den (absoluten) Unterschieden in den Attributen von Personen oder Positionen, sondern entsteht aus den sozialen Beziehungen zwischen ihnen. Die Autoren konzeptualisieren den Lohnbildungsprozess daher als Aushandlungsprozess zwischen (kollektiven) Akteuren um die vor Ort angemessene oder als legitim geltende Verteilung der im Betrieb vorhandenen Geldmengen (TomaskovicDevey, 2014). Sie bezeichnen diesen Prozess, bei dem Akteure um die Zuteilung von Geldmengen kämpfen, als claims-making. Dieses besteht aus zwei Stufen (Avent-Holt \& Tomaskovic-Devey, 2014, S. 384): Zunächst erhebt ein Akteur Anspruch. Dies kann expli- 
zit geschehen wie die Nachfrage nach einer Beförderung oder Gehaltserhöhung oder das Anstrengen von Tarifverhandlungen im Fall von Gewerkschaften. Es können aber auch Änderungen einer impliziten, selbstverständlichen Praxis sein (des Budgets einer Abteilung oder des Grundgehalts eines Jobs). Dem Anspruch wird dann im zweiten Schritt durch geeignete Mittel und Ressourcen Nachdruck verliehen. Dabei geht es vor allem darum, relevanten Entscheidern aufzuzeigen, warum der eigene Anspruch legitimer ist als derjenige anderer Akteure. Gelingt dies, werden die relevanten Entscheider dieser Forderung eher entsprechen und die geforderten Geldmengen zuteilen. An beiden Stufen des claimsmakings wird Ungleichheit generiert: Statusniedrigere Gruppen erheben seltener Anspruch und diesem wird weniger häufig entsprochen. Im folgenden Abschnitt werden zwei Ressourcen besprochen, die diesen Erfolg beeinflussen.

\subsection{Kategorien und betriebliche Umwelt als Ressourcen im claims- making}

Den Akteuren stehen im claims-making vor allem zwei Ressourcen zur Verfügung: Kategorien und die betriebliche Umwelt. Kategorien bilden Grenzen. Sie teilen die Akteure im Betrieb in Gruppen ein, sodass einige dazu gehören und andere nicht. Einige Kategorien sind nur lokal, in diesem Betrieb gültig. Sie entstehen aus dem Produktionsprozess und der lokal implementierten Arbeitsteilung (Eigentümer-Arbeiter, Fließbandarbeiter-Meister). Andere kategoriale Unterscheidungen haben eine externe, soziale Gültigkeit (Mann-Frau, Hochschulabschluss-kein Hochschulabschluss). Akteure nutzen diese Gruppenzugehörigkeit, um im interaktionalen Prozess des claims-makings ihren Anspruch durchzusetzen. ${ }^{2}$ Kategorien aktivieren in Interaktionen Erwartungen, Klischees, Vorurteile und Stereotypen. Diese Erwartungen können im Arbeitskontext genutzt werden um Statushierarchien zu aktivieren und so den Anspruch des einen Akteurs gerechtfertigter erscheinen zu lassen als den eines anderen (Tomaskovic-Devey, 2014). Im Folgenden verwende ich daher auch den Begriff „Statuskategorie“. Gerade die Geschlechtskategorie ist in quasi jeder Interaktion eine bedeutsame Statuskategorie und daher auch (und gerade) im Arbeitskontext wirksam im Hinblick auf das Einkommen (Gorman, 2005; Moss \& Tilly, 2003; Ridgeway, 1997; Roscigno, Garcia \& Bobbitt-Zeher, 2007). Die größte legitimatorische Wirkung erzielen Kategorien dann, wenn sie sich überschneiden. Insbesondere da, wo sich betriebsinterne Kategorien mit externen, kulturell geprägten Kategorien (Männer im Management vs. weibliche Arbeiter, Männer in Vollzeit vs. Frauen in Teilzeit, etc.) verbinden, sollte das Ausmaß der Ungleichheit am größten sein. Betriebe bilden spezifische Verläufe von Kategoriengrenzen und Relationen zwischen Statuskategorien (hier als „Statusrelationen“ bezeichnet) aus (Avent-Holt \& Tomaskovic-Devey, 2014, S. 386). Es sind diese betriebsspezifischen Statusrelationen entlang der Geschlechtskategorie, die das Ausmaß des GWG im Betrieb beeinflussen.

2 Bildung ist in diesem Modell ebenfalls eine solche Kategorie: Akteur A hat Qualifikation X und es ist daher gerechtfertigt, dass er einen höheren Lohn erhält als Akteur B. Dies spiegelt aber nicht die höhere Produktivität der Arbeitskraft wider, sondern ist Resultat des claims-makings, in dem Bildung als Ressource eingesetzt werden kann, um den Anspruch des Akteurs gegenüber anderen Akteuren zu legitimieren. 
Neben sozialen Kategorien kann auch die betriebliche Umwelt eine Ressource bei der Durchsetzung von Ansprüchen sein. Institutionelle Umwelten formulieren Regeln für das angemessene Verhalten von Akteuren. Dies kann einerseits auf der Ebene der Organisation selbst eine Rolle spielen. Unternehmen (insbesondere große und damit für die Öffentlichkeit sichtbare) müssen die Legitimität der organisationalen Entscheidungen und Praktiken gegenüber anderen Akteuren sicherstellen. Aber auch für die interne Arbeitsorganisation (Arbeitsteilung, Qualifikationsvoraussetzungen für bestimmte Jobs, Grundgehälter für bestimmte Positionen) gibt es Regeln und Erwartungen, die von Akteuren (Gewerkschaften, Berufsverbände, Gesetze) in diesem Feld formuliert werden. All diese Regeln beeinflussen das claims-making im Betrieb, indem sie den Anspruch des einen Akteurs eher stützen, während der von anderen entkräftet wird.

Das claims-making stellt nun die interaktionale Grundlage für einen zweiten Mechanismus dar, mit dem der Zugang zu ,guten“ Positionen und Jobs im Betrieb nur für bestimmte Akteure möglich wird (,Chancenhortung“).

\subsection{Chancenhortung}

Betriebe bilden Positionshierarchien aus, sodass höhere Positionen mit einer höheren Entlohnung verbunden sind. Lohnungleichheit kann hergestellt werden, indem Positionsinhaber den Zugang für bestimmte Akteure beschränken, während er für andere Akteure (Personen aus dem eigenen Netzwerk, Freunde, bestimmte Bildungstitel, kategorial ähnliche Personen) ermöglicht wird. Im Rahmen der RIT werden diese Prozesse als „opportunity hoarding“ (Chancenhortung) bezeichnet. ${ }^{3}$ Dieses Matching von Personen zu Positionen im Betrieb ist ein zentraler Mechanismus der Herstellung von (geschlechtsspezifischer) Lohnungleichheit. Chancenhortung setzt auf dem claims-making auf, das diesen ungleichen $\mathrm{Zu}$ gang zu Positionen im Betrieb legitimiert. Insofern werden Erfolge im claims-making teilweise über den Prozess der Chancenhortung in Lohnungleichheiten übersetzt.

Zahlreiche nationale wie auch internationale Studien weisen auf den Zusammenhang von Jobsegregation und GWG hin. ${ }^{4}$ Studien für Deutschland zeigen, dass die Jobsegregation innerhalb von Betrieben gemessen am Duncan-Index bei über $70 \%$ liegt (Projektgruppe GiB, 2010, S. 116). Gartner und Hinz (2009) zeigen ebenfalls, dass der GWG innerhalb von Jobs kleiner ist als im ganzen Betrieb - sich allerdings mit etwa 85\% im Jahr 2006 im Vergleich zu den USA oder Schweden auf einem relativ hohen Niveau bewegt. Die geschlechtsspezifische Zuweisung von Personen zu Positionen ist also einer der zentralen Mechanismen der Herstellung von Lohnungleichheit.

3 Chancenhortung ist eng verbunden mit dem Konzept der sozialen Schließung. Folgt man Diewald und Faist (2011), bezeichnet Chancenhortung Schließungsprozesse innerhalb von Organisationen (im Unterschied zur Exklusion, die Schließungsprozesse zwischen Organisationen beschreibt).

4 Als aktuelle internationale Studie sei hier Cardoso, Guimarães und Portugal (2016) erwähnt. Die Autoren zeigen mit einem sehr informationsreichen LEED für Portugal, dass die Segregation von Frauen in niedrigentlohnenden Betrieben und Jobs einen großen Teil des GWG erklärt. 


\section{Hypothesen und Forschungsstand}

Der Ausgang des claims-makings wird von den Statuskategorien beeinflusst, die den beteiligten Akteuren zur Verfügung stehen. Das Ausmaß der geschlechtsspezifischen Lohnungleichheit hängt folglich vom Unterschied zwischen den Geschlechtern in der Kontrolle dieser Statuskategorien im Betrieb ab. Im Folgenden werden nun zwei zentrale betriebliche Statusrelationen vorgestellt, die je eine betriebsinterne (Managementposition) und eine kulturelle (Bildungstitel) Statuskategorie aufgreifen, und deren Einfluss auf den relativen Lohn von Frauen aufgezeigt.

\subsection{Frauenanteil im Management}

Personen in Managementpositionen gelten als Leistungsträger und genießen daher eine hohe Autorität. Wenn der Anteil an Frauen in diesen Positionen im Vergleich zu Männern steigt, sollte die Gültigkeit von Frauen als untergeordnete Statusgruppe abnehmen (Tomaskovic-Devey, Hällsten \& Avent-Holt, 2015). Diese Aufwertung des Status der Kategorie „Frau“ gilt betriebsweit und sollte daher die Lohnansprüche aller Frauen im Betrieb verbessern - unabhängig von ihren individuellen Merkmalen. Zudem können Frauen im Management auch einen direkteren Einfluss ausüben, indem sie als Verbündete fungieren, um anderen Frauen zu helfen, ihre Ansprüche gegenüber männlichen Kollegen durchzusetzen. Männliche Manager bevorzugen häufig (bewusst oder unbewusst) ihnen ähnliche Bewerber bei der Besetzung von Positionen (homosoziale Rekrutierung), wodurch Frauen auch bei gleicher Eignung für die Stelle systematisch benachteiligt werden (Broadbridge \& Hearn, 2008; Castilla, 2011; Holgersson, 2013). Ein höherer Frauenanteil im Management sollte daher einerseits dieses homosoziale Rekrutierungsverhalten von Männern beschränken. Zudem können weibliche Manager ein ähnliches Verhalten aufweisen und systematisch weibliche Bewerber bevorzugen (Kunze \& Miller, 2014).

\section{H1: Je höher der Frauenanteil im Management von Betrieben, desto höher der relative Lohn von Frauen.}

Eine Reihe von internationalen Studien zeigt, dass Betriebe, in denen Frauen stark im Management repräsentiert sind, einen geringeren GWG und eine höhere Beförderungswahrscheinlichkeit von Frauen in mittlere und gehobene Managementpositionen aufweisen (Schweden: Hensvik, 2014; Hultin \& Szulkin, 1999, 2003. Portugal: Cardoso \& WinterEbmer, 2010. USA: Cohen \& Huffman, 2007; Huffman, Cohen \& Pearlman, 2010; Kurtulus \& Tomaskovic-Devey, 2012). Die Studie von Hirsch (2013) ist eine der wenigen Studien, die diesen Zusammenhang bisher für Deutschland untersucht hat. Auch sie kommt mit den LIAB-Daten von 2008 zum Ergebnis, dass ein höherer Frauenanteil im Management den GWG im Betrieb (genauer: in Jobzellen) reduziert.

\subsection{Geschlechterunterschiede bei den Bildungstiteln}

Bildungstitel (Hochschulabschlüsse, Meister) beeinflussen Löhne nicht nur in Form von Produktivität und als Zertifikat für den Zugang zu bestimmten Positionen, sie verändern auch die Statushierarchie im Betrieb zugunsten der Träger dieser Titel, da der Besitz von Bildungsti- 
teln mit einem höheren Status assoziiert ist. Wenn sich Bildungstitel mit anderen positiv assoziierten Merkmalen wie dem männlichen Geschlecht überlappen, entstehen entlang dieser Statusrelation besonders große Lohnungleichheiten. Entsprechend sollte sich der Status aller Frauen im Betrieb (und damit die relativen Löhne aufgrund der besseren Position im claimsmaking) im Vergleich zu Männern erhöhen, wenn die Vorteile bei den Bildungstiteln anwachsen. Frauen haben also einen Vorteil, wenn sie in Betrieben arbeiten, in denen Frauen im Vergleich zu ihren männlichen Kollegen beispielsweise einen höheren Anteil an Hochschulabschlüssen aufweisen - unabhängig von ihrem eigenen Bildungsstand. Tomaskovic-Devey und Coautoren (2015) zeigen für Schweden, dass die Lohnungleichheit zwischen Einheimischen und Immigranten abnimmt, wenn die Bildungsvorteile der Immigranten steigen.

H2: Je größer die Bildungsvorteile von Frauen im Vergleich zu Männern im Betrieb, desto höher der relative Lohn von Frauen.

Die vorgestellten Statuskategorien stellen also zwei Möglichkeiten dar, um den relativen Status und damit die Legitimität der Ansprüche von Frauen im claims-making im Vergleich zu Männern im Betrieb zu konzeptualisieren. Je nach betrieblicher Umwelt können sich diese Statusrelationen aber mal mehr, mal weniger auf das claims-making auswirken. Im folgenden Abschnitt wird mit der Tarifbindung ein solcher Umwelteinfluss besprochen.

\subsection{Betriebliche Umwelt: Tarifbindung des Betriebs}

Obwohl auch in Deutschland ein dramatischer Rückgang der Tarifbindung in den letzten Jahren zu beobachten ist, sind immer noch viele Arbeitsverhältnisse durch zentral für eine ganze Branche (Flächentarifvertrag) ausgehandelte Löhne bestimmt. Anhand dieser Differenzierung nach Tarifbindung soll ein zusätzlicher Prüfstein für die RIT eingeführt werden. Die ungleichheitsgenerierende Wirkung von Statusunterschieden entlang der Geschlechtskategorie sollte in tarifgebundenen Betrieben abgeschwächt sein, da lokale, im Betrieb gültige Statusrelationen ein geringeres Gewicht bei der Bestimmung von Löhnen haben. Zentrale Lohnverhandlungen auf Brancheneben lassen weniger Spielraum für die betriebsspezifische Etablierung von Lohnungleichheiten entlang der Geschlechtskategorie.

H3: Der Einfluss betrieblicher Statusrelationen auf den GWG sollte unter Tarifbindung schwächer sein.

\subsection{Unterschiede entlang der Lohnverteilung}

Eine Reihe aktueller Studien zeigt übereinstimmend, dass der GWG entlang der Lohnverteilung variiert (Arulampalam, Booth \& Bryan, 2007; Kassenboehmer \& Sinning, 2014). Dabei zeigt sich zumeist eine Verbreiterung der Lohnlücke in den oberen Regionen der Verteilung. Dieses Phänomen wird häufig als gläserne Decke (glass ceiling) bezeichnet. Diese Studien werfen die Frage auf, ob die bisher identifizierten Statusrelationen eine unterschiedliche Wirkung an verschiedenen Stellen der Lohnverteilung (d.h. bei unterschiedlichen Lohnniveaus) haben.

Sowohl ein steigender Frauenanteil im Management als auch steigende Bildungsvorteile von Frauen relativ zu Männern erhöhen den Status der Kategorie Frau. Dies geschieht vor allem über die Zuschreibung von Leistungsfähigkeit und Kompetenz, die Managementpositio- 
nen wie auch Bildungstitel signalisieren. Da hohe Lohnansprüche häufig mit genau solchen Argumenten legitimiert werden, sollte der Einfluss auf hohe Löhne größer sein als im unteren Bereich der Lohnverteilung. Durch die zunehmende Verbreitung von Bonuszahlungen am oberen Ende der Lohnverteilung wird dieses Argumentationsmuster zunehmend wichtiger (Lemieux, MacLeod \& Parent, 2009; für Deutschland: Sommerfeld, 2013) - gerade in Betrieben ohne Tarifbindung. Beim Frauenanteil im Management kommt hinzu, dass es Frauen auf Ebenen unter dem Management aufgrund des reduzierten homosozialen Rekrutierungsverhaltens von Männern leichter fallen sollte, in höhere Positionen zu gelangen, sodass auch hier zu erwarten ist, dass der Effekt im oberen Teil der Lohnverteilung stärker ausfällt.

\section{H4: Der positive Einfluss des Frauenanteils im Management auf die relativen Löhne von Frauen nimmt entlang der Lohnverteilung zu.}

H5: Der positive Einfluss von Bildungsvorteilen auf die relativen Löhne von Frauen nimmt entlang der Lohnverteilung zu.

Insgesamt zeigt die vorherige Diskussion, dass die Implikationen der RIT bisher vergleichsweise wenig mit empirischen Studien überprüft wurden. Am häufigsten wurde in vorwiegend internationalen - Studien der Einfluss des Frauenanteils im Management auf den relativen Lohn der Frauen untersucht. Andere Indikatoren und insbesondere deren Variation nach Tarifbindung und Lohnniveau wurden bislang kaum erforscht. Die vorliegende Studie versucht hier einen Beitrag zu leisten, indem zum einen überhaupt die RIT dazu verwendet wird, empirische Forschung anzuleiten, und zum anderen die zentralen Implikationen des Modells am Beispiel Deutschland überprüft werden.

\section{Daten und Analysemethoden}

\subsection{Daten}

Die Analysen basieren auf der Verdienststrukturerhebung (VSE) 2010. ${ }^{5}$ Die VSE ist eine vom Statistischen Bundesamt bereitgestellte zweistufige Querschnittsstichprobe: Auf der ersten Ebene werden Betriebe aus dem Unternehmensregister gezogen. Auf der zweiten Ebene werden Beschäftigte aus diesen Betrieben ausgewählt. So entsteht ein Datensatz, in dem die Personen zusammen mit ihren jeweiligen Betrieben beobachtet werden (linked employer-employee data, LEED). ${ }^{6}$ Die Auswahlgrundlage umfasst Betriebe mit mindestens zehn sozialversicherungspflichtigen Beschäftigten aus allen Wirtschaftsbereichen.

Die VSE hat eine Reihe von Vorteilen: Erstens besitzt sie einen großen Stichprobenumfang. Für das Jahr 2010 stehen fast zwei Millionen Beobachtungen auf der Personenebene und über 30.000 Beobachtungen auf der Betriebsebene zur Verfügung. Zweitens beinhaltet die VSE eine sehr genaue Messung des Lohns, die zudem nicht zensiert ist. Die Arbeitgeber haben eine Auskunftspflicht, was die Qualität der Daten im Vergleich zu freiwilligen Haushaltssurveys erhöhen dürfte. Damit eignet sich die VSE für detaillierte Verdienstanalysen - insbesondere auch am oberen Ende der Lohnverteilung. Schließlich wird

5 Weiter Informationen zur VSE finden sich in Günther (2013).

6 Es werden entsprechende Gewichte bei allen Analysen verwendet, um die unterschiedliche Ziehungswahrscheinlichkeit durch die geclusterte und geschichtete Stichprobe auszugleichen. 
die Arbeitszeit in Stunden erhoben, was eine Analyse von Stundenlöhnen und damit den Einbezug von Teilzeitbeschäftigten ermöglicht. Diese letzten beiden Vorteile in Verbindung mit den detailliert erhobenen Bonuszahlungen legen die Nutzung der VSE im Gegensatz zum LIAB des IAB, der zweiten bekannten Datenquelle in Deutschland, nahe - insbesondere, wenn es um die Analyse der oberen und unteren Ränder der Lohnverteilung geht. ${ }^{7}$

Aus dem vollen Datenbestand werden Beamte, Heimarbeiter, Auszubildende, Personen in Altersteilzeit und Personen in marginaler Beschäftigung (Arbeitszeit von weniger als 18 Stunden in der Woche) entfernt. Das Analysesample beschränkt sich damit auf Erwerbstätige im Alter zwischen 16 und 65 mit einer wöchentlichen Arbeitszeit von mindestens 18 Stunden. Es werden zudem nur solche Betriebe berücksichtigt, die mindestens fünf Frauen und fünf Männer beschäftigen und die wenigstens eine Managementposition aufweisen. Diese Bedingung ist notwendig, damit den pro Betrieb berechneten Statusrelationen eine ausreichende Datenbasis zugrunde liegt. Diese Selektionsentscheidung führt dazu, dass kleine Betriebe tendenziell unterrepräsentiert sind. ${ }^{8}$ Darüber hinaus sind Betriebe aus dem produzierenden Gewerbe leicht überrepräsentiert. Insgesamt sind die Unterschiede allerdings gering, insbesondere die Branchenzusammensetzung ändert sich kaum. Die Analysen werden getrennt für West- und Ostdeutschland durchgeführt, wobei der Fokus der Analyse auf den alten Bundesländern liegt.

\subsection{Variablen}

Die abhängige Variable ist der Bruttostundenlohn in Euro. Er ergibt sich aus der Division des Bruttomonatsverdiensts (inklusive regelmäßiger monatlicher sowie unregelmäßiger Sonderzahlungen zum Jahresende) und der monatlich tatsächlich geleisteten Arbeitszeit (inklusive bezahlter Mehrarbeitsstunden). ${ }^{9}$ Eine Logarithmierung korrigiert die rechtsschiefe Verteilung des Bruttostundenlohns.

Die theoretischen Ausführungen legen nahe, dass betriebsspezifische Statusrelationen die geschlechtsspezifische Lohnungleichheit beeinflussen. Entsprechend werden entlang der Geschlechtskategorie zwei Indikatoren konstruiert. Dabei geht es nicht um den absoluten Besitz von Statuskategorien, sondern um Relationen zwischen den Geschlechtern. Diesem Umstand wird bei der Operationalisierung Rechnung getragen, indem für jeden Betrieb Differenzen oder Anteile von Statuskategorien berechnet werden, die auf die beiden Geschlechter entfallen.

Für den Frauenanteil im Management wird auf die Angabe der „Leistungsgruppe“ zurückgegriffen. Arbeitgeber müssen MitarbeiterInnen in eine von fünf Leistungsgruppen

7 Der LIAB hat durch seine Panelstruktur und reicheren Informationen auf der Betriebsebene andere Vorteile, die für die vorliegende Studie aber nicht entscheidend sind. Vielmehr könnte man das Panel in einem zweiten Schritt nutzen und die hier gefundenen Ergebnisse überprüfen.

8 Das arithmetische Mittel der Betriebsgröße steigt durch diese Selektion von 1.194 auf 1.496 Personen pro Betrieb. Der GWG (berechnet als Differenz des logarithmierten Bruttostundenlohns) erhöht sich von 0,215 $(0,038)$ im Westen (Osten) auf 0,254 (0,137) Logpunkte durch diese Selektion und wird daher vor allem für Ostdeutschland leicht überschätzt.

9 Aktuelle Studien zeigen, dass Sonderzahlungen gerade am oberen Ende der Lohnverteilung einen großen Einfluss auf die Lohnungleichheit haben (Lemieux, MacLeod \& Parent, 2009; Schweiker \& Groß, 2016). Es ist eine Stärke der VSE, dass durch die Verfügbarkeit dieser Sonderzahlungen das gesamte Ausmaß des GWG besser erfasst werden kann. 
einordnen. Dabei handelt es sich um lokal gültige Rangunterschiede, die insbesondere Unterschiede in der Aufsichts- und Dispositionsbefugnis und der Komplexität der Tätigkeiten widerspiegeln. Leistungsgruppe 1 „Arbeitnehmer/innen in leitender Stellung mit Aufsichtsund Dispositionsbefugnis“ wird als Management definiert. ${ }^{10}$ Für jeden Betrieb wird dann der Anteil an Frauen in diesen Positionen der Leistungsgruppe 1 berechnet. Für die Operationalisierung von Bildungsvorteilen wird auf die Information über den Besitz eines Hochschulabschlusses zurückgegriffen. Die betriebliche Statusrelation wird als Differenz im Anteil an Hochschulabschlüssen zwischen den Geschlechtern pro Betrieb berechnet. ${ }^{11}$

Neben diesen zwei zentralen Betriebsmerkmalen werden zahlreiche Kontrollvariablen verwendet. Dies schließt zum einen typische allgemeine und spezifische Humankapitalfaktoren auf der Individualebene ein. Es wird für Betriebszugehörigkeitsdauer, Alter in Jahren, Bildungsjahre und Teilzeitbeschäftigung kontrolliert. Zum anderen werden Jobmerkmale wie ein befristeter Arbeitsvertrag und der berufliche Rang miteinbezogen. Letzterer wird verwendet, um zumindest näherungsweise die betriebliche Jobstruktur abzubilden, da davon ausgegangen wird, dass die Zuweisung von Personen zu unterschiedlichen Jobs ein zentraler Mechanismus der Herstellung des GWG ist. Für die Konstruktion des beruflichen Ranges wird der 3-Steller der Klassifikation der Berufe (KldB 88) verwendet und ein Ranking anhand des jeweiligen Durchschnittseinkommens erstellt. Der Indikator hat einen Wertebereich von 0 bis 1, wobei der Wert ausdrückt, welcher Anteil an Berufen ein geringeres Durchschnittseinkommen aufweist als der betreffende Beruf (für eine genauere Beschreibung siehe Tomaskovic-Devey et al. 2015).

Daneben werden zahlreiche Kontrollvariablen auf der Betriebsebene verwendet: durchschnittliche Bildungsjahre der Mitarbeiter im Betrieb, durchschnittliche Betriebszugehörigkeitsdauer, Anteil Frauen im Betrieb, Betriebsgröße und eine Reihe von Branchenindikatoren. Schließlich stehen auch Informationen darüber zur Verfügung, ob ein Tarifvertrag im Betrieb Anwendung findet. Es wird zwischen Betrieben mit Branchentarifbindung und Betrieben, die auf individuelle Lohnverhandlungen setzen, unterschieden.

\subsection{Analysestrategie}

Die bisherigen Überlegungen implizieren einen zwischen den Betrieben variierenden GWG, der mit betriebsspezifischen Statusrelationen zusammenhängt. Damit sind primär nicht Personenmerkmale von Interesse, sondern Interaktionen zwischen dem Geschlecht und Betriebsmerkmalen. Ausgehend hiervon bieten sich Multilevel Modelle für die Analyse an (Rabe-Hesketh \& Skrondal, 2012). Genauer wird ein Random-Intercept-Random-SlopeModell mit zwei cross-level-Interaktionen geschätzt. Es kann wie folgt beschrieben werden:

$$
\begin{gathered}
\log \left(\text { Lohn }_{i j}\right)=\left(\beta_{1}+\varsigma_{1 j}\right)+\left(\beta_{2}+\varsigma_{2 j}\right) \text { Frau }_{i j}+\beta_{3} \text { Frau } * \% \text { FrauManagement }+ \\
\beta_{4} \text { Frau } * \text { DiffHochschule }+\cdots+\beta_{p} x_{p i j}+\beta_{p} w_{p j}+\epsilon_{i j},
\end{gathered}
$$

10 Mit den vorhandenen Informationen ist es allerdings nicht möglich, das Top-Management trennscharf von mittleren oder gehobenen Managementpositionen abzugrenzen. Insofern befinden sich in Leistungsgruppe 1 nicht nur Geschäftsführer und Vorstände, sondern bspw. auch Abteilungs- oder Ressortleiter.

11 Es wurde darüber hinaus eine alternative Operationalisierung getestet, wobei pro Betrieb der Korrelationskoeffizient zwischen Geschlecht und Hochschulabschluss berechnet wurde. Die Ergebnisse unterscheiden sich kaum. 
wobei $\log \left(L o h n_{i j}\right)$ der logarithmierte Bruttostundenlohn von Mitarbeiter $i$ in Betrieb $j$ ist und die Terme $\varsigma_{j}$ und $\epsilon_{i j}$ betriebsspezifische respektive personenspezifische Fehlerterme darstellen. Der Term $\varsigma_{2 j}$ bezeichnet die Abweichung des Koeffizienten für Frauen zwischen Betrieben vom mittleren Koeffizienten. ${ }^{12}$

In einem ersten Schritt werden diese Modelle für deskriptive Zwecke verwendet. Zunächst wird ein Modell geschätzt, in das nur ein Indikator für Frauen und alle Kontrollvariablen auf der Individualebene aufgenommen werden. Damit kann gezeigt werden, wie stark der Effekt des Frauenindikators (und damit des GWG) zwischen Betrieben variiert - nachdem für individuelle Unterschiede im Humankapital kontrolliert wurde.

Im zweiten Schritt werden den Modellen cross-level Interactionen zwischen dem Frauenindikator und den zwei betrieblichen Statusrelationen sowie dem Frauenanteil im Betrieb hinzugefügt. Die geschätzten Koeffizienten geben an, inwiefern sich der durchschnittliche Bruttostundenlohn von Frauen in bestimmten betrieblichen Kontexten verändert und liefern damit Tests für die Hypothesen H1-H2. Die Modelle werden in drei Schritten aufgebaut: M1 enthält die Interaktionen und alle Haupteffekte sowie die bereits erläuterten Personenund Betriebskontrollen - mit Ausnahme des individuellen beruflichen Rangs. Diese Variable wird in M2 hinzugefügt. Ein zentraler Mechanismus der Herstellung von Lohnungleichheit im Betrieb ist die Zuweisung zu unterschiedlichen Jobs (Chancenhortung). Die betrieblichen Statusrelationen sollten sich zumindest teilweise über diese Schließungsprozesse auf den relativen Lohn der Frauen auswirken (Mediatoreffekt). In M3 werden schließlich noch weitere Interaktionen zwischen dem Geschlecht und individuellen Merkmalen wie Bildung, Betriebszugehörigkeitsdauer und beruflicher Rang hinzugefügt, um zu kontrollieren, ob sich der Einfluss dieser individuellen Ressourcen auf den Bruttostundenlohn zwischen den Geschlechtern unterscheidet. Um zu überprüfen, ob der Einfluss der Statusrelationen in tarifungebundenen Betrieben höher ist (H3), werden die Modelle ferner getrennt für Betriebe mit individuellen Lohnverhandlungen und mit Tarifbindung geschätzt. Schließlich werden alle Modelle getrennt für Ost- und Westdeutschland berechnet.

Um Effekte nicht nur auf den Mittelwert, sondern auf beliebige Quantile der Verteilung zu erhalten, werden im zweiten Analyseteil Quantilsregressionen berechnet (H4-H5). Ein Problem von Standardquantilsregressionen ist allerdings, dass sich die Koeffizienten nur als Einfluss auf das bedingte Quantil interpretieren lassen. Gerade bei Ungleichheitsanalysen ist aber weniger der bedingte (d.h. der Effekt innerhalb von Gruppen definiert durch die Kovariablen) als vielmehr der marginale Effekt von Interesse, da dieser den Einfluss auf die unbedingte Lohnverteilung der Geschlechter anzeigt und somit direkt Aussagen über die Veränderung der GWG erlaubt. Firpo, Fortin und Lemieux (2009) stellen in einem neuen Ansatz eine Möglichkeit vor, wie man die Einflüsse von Kovariablen auf Quantile der unbedingten Lohnverteilung schätzen kann. Sie greifen dabei auf ein Konzept aus der robusten Statistik zurück: die Einflussfunktion (recentered influence function, RIF). Die Autoren zeigen, dass die geschätzten Koeffizienten einer OLS-Regression auf diese RIF-Werte als Einfluss auf das marginale (unbedingte) Quantil interpretiert werden können. ${ }^{13}$

12 Die Modelle wurden mit Stata 13 und dem Befehl ,xtmixed logLohn X || BetriebsID: Frau, cov(unstructured)“"geschätzt.

13 Die Modelle wurden mit Stata 13 unter Verwendung des ados ,rifreg“, das von Nicole Fortin auf ihrer Website bereitgestellt wird, berechnet. 


\section{Ergebnisse}

Es werden zunächst in Abschnitt 5.1 die Variation des GWG zwischen Betrieben und entlang der Lohnverteilung dargestellt. In Abschnitt 5.2 werden die Ergebnisse der Multilevel Modelle und in Abschnitt 5.3 die der Quantilsregressionen vorgestellt.

\subsection{Die Variation der geschlechtsspezifischen Lohnungleichheit zwischen Betrieben und entlang der Verteilung}

Folgt man der Argumentation der RIT, sollte der GWG zwischen Betrieben variieren, da die jeweiligen betrieblichen Kontexte die individuellen Lohnchancen beeinflussen. Abbildung 1 stellt die Dichtefunktion der geschätzten random effects des Frauenindikators getrennt für Ost- und Westdeutschland dar. Deutlich zu sehen ist, dass der Mittelwert dieser Verteilung für beide Landesteile im negativen Bereich liegt. Im Schnitt verdienen Frauen also im Betrieb weniger als ihre männlichen Kollegen - nach Kontrolle relevanter Humankapitalmerkmale. Es zeigt sich ebenfalls, dass der GWG in den alten Bundesländern stärker ausgeprägt ist als in den neuen. In einigen Betrieben werden allerdings auch positive Koeffizienten geschätzt. Dort verdienen Frauen also mehr.

Abbildung 1: Variation des GWG zwischen Betrieben getrennt für Ost- und Westdeutschland

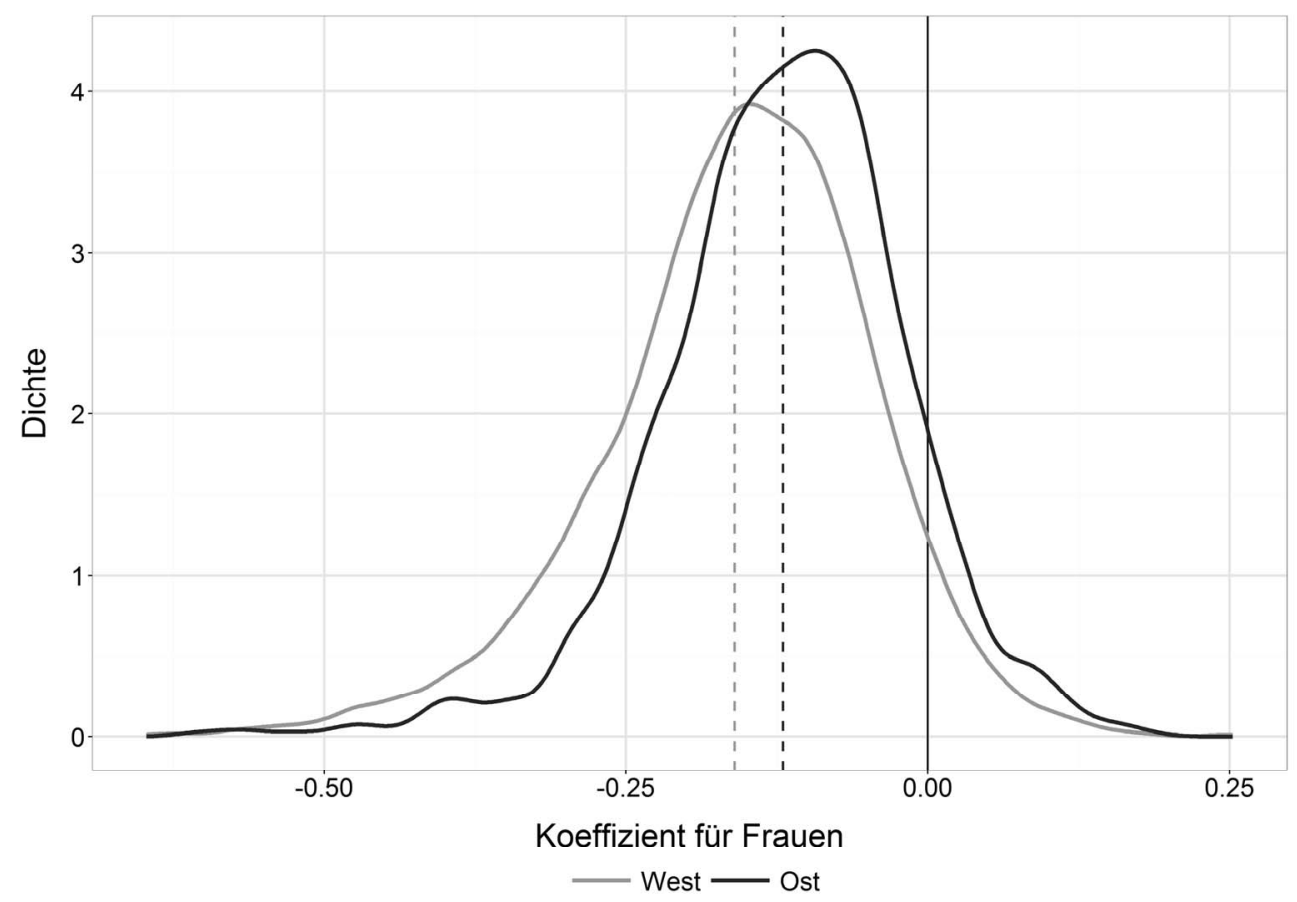

Anmerkung: Dargestellt ist die Dichteverteilung des Koeffizienten für Frauen über alle Betriebe aus einem random coefficient model mit Kontrollvariablen auf der Personenebene. 
Neben der Variation des GWG zwischen Betrieben zeigen aktuelle Studien auch eine Variation entlang der Lohnverteilung. Bisher liegen für Deutschland noch keine Studien vor, die den GWG entlang der gesamten Verteilung darstellen und dabei sowohl Teilzeitbeschäftigung als auch Sonderzahlungen berücksichtigen. Abbildung 2 stellt Quantilsdifferenzen im logarithmierten Bruttostundenlohn für das 1. bis 99. Perzentil und die Unterschiede im mittleren Lohn (horizontale Linie) für die beiden Landesteile dar. Zunächst zeigt sich der bekannte Unterschied im mittleren GWG. Dieser liegt in Westdeutschland bei 0,25 Logpunkten und ist damit deutlich größer als in Ostdeutschland $(0,14)$. Unterschiede im Mittelwert verdecken aber die ausgeprägte Variation des GWG entlang der Verteilung. Für Westdeutschland beträgt er bis zum 25. Perzentil unter 0,2 Logpunkte, danach steigt er auf über 0,5 Logpunkte am 99. Perzentil. Damit können mit der VSE die Ergebnisse von Arulampalam und Coautoren (2007) untermauert werden, wonach es auch in Deutschland (neben vielen anderen europäischen Ländern und den USA) eine „glass ceiling“ zu geben scheint. Nach unten hin (vor allem zwischen dem 1. und 5. Perzentil) nimmt der GWG dagegen deutlich ab. Die Kurve für Ostdeutschland folgt diesem generellen Trend, zeigt aber einen deutlichen Bauch in der Mitte der Verteilung. Im Osten steigt der GWG also weniger linear an, sondern nimmt zum Median hin zunächst ab, um dann stark anzusteigen.

Abbildung 2: Variation des GWG entlang der Lohnverteilung getrennt für Ost- und Westdeutschland

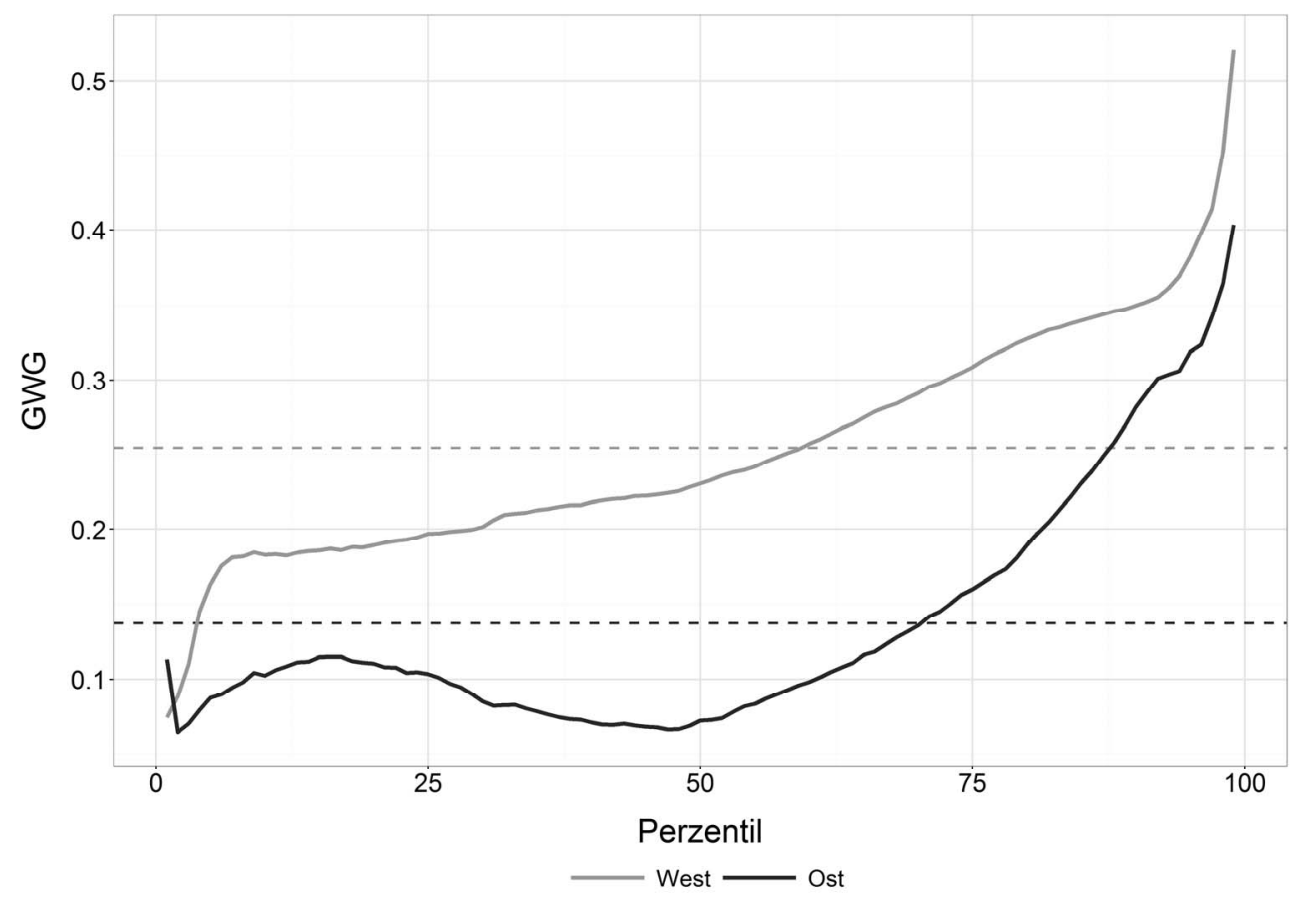

Anmerkung: GWG berechnet als samplegewichtete Differenz zwischen dem arithmetischen Mittel bzw. dem jeweiligen Perzentil des log. Bruttostundenlohns der Männer und demjenigen der Frauen. 
Insgesamt zeigt sich also, dass der GWG sowohl zwischen Betrieben als auch entlang der Lohnverteilung variiert. Die folgenden multivariaten Analysen versuchen nun diese Variation mit den betrieblichen Statusrelationen zu erklären.

\subsection{Der Einfluss betrieblicher Statusrelationen auf die mittleren relativen Löhne von Frauen}

Im Folgenden werden die Ergebnisse der Multilevel Modelle für Westdeutschland präsentiert (Tabelle 1). Hypothese 1, wonach sich der relative Lohn von Frauen mit dem Anteil von Frauen im Management erhöht, findet Unterstützung. Der Koeffizient des Interaktionseffekts ist positiv und hochsignifikant. Dieses Resultat bestätigt Ergebnisse aus der Literatur, die ebenfalls einen positiven Effekt finden (Hirsch, 2013; Hultin \& Szulkin, 2003; Kurtulus \& Tomaskovic-Devey, 2012). Mit steigendem Frauenanteil im Management erhöht sich der Status von Frauen im Betrieb und damit die Durchsetzungskraft von Ansprüchen im claims-making, was sich schließlich in höheren relativen Löhnen von Frauen in solchen Betrieben zeigt. Der Effekt ist ferner signifikant stärker in Betrieben ohne Tarifbindung, da sich hier Statusrelationen stärker auf die Verteilung der Löhne zwischen den Geschlechtern auswirken können. In Modell 2 wird der berufliche Rang in das Modell aufgenommen, um zu überprüfen, ob sich ein wachsender Frauenanteil in einen besseren $\mathrm{Zu}$ gang zu höheren Positionen übersetzt. Der Effekt sinkt in Modell 2 zwar leicht, bleibt insgesamt aber relativ stabil. Ein höherer Frauenanteil wirkt sich also nur geringfügig über den besseren Zugang zu Positionen auf den Lohn aus. Vielmehr scheinen Frauen innerhalb von Positionen einen Vorteil zu haben, wenn der Frauenanteil im Management steigt. Gerade in Deutschland, wo der GWG im gleichen Job vergleichsweise hoch ist (Gartner \& Hinz, 2009), könnten Betriebe, in denen der Frauenanteil im Management hoch ist, eine Antidiskriminierungspolitik fahren und so Benachteiligungen von Frauen gerade auch innerhalb von Jobs reduzieren. Die Ergebnisse für Ostdeutschland unterscheiden sich kaum von den hier beschriebenen.

Das Diskriminierungsmodell von Becker könnte diese Ergebnisse ebenfalls erklären, wenn man davon ausgeht, dass diskriminierende Arbeitgeber weniger Frauen im Management beschäftigen und gleichzeitig alle Frauen im Betrieb geringer entlohnen. Gegen diese Interpretation gibt es zwei Hinweise: Zum einen kann der Frauenanteil im Betrieb als Proxy für Diskriminierungspräferenz aufgefasst werden, welche damit kontrolliert ist. ${ }^{14} \mathrm{Zum}$ anderen zeigt die Interaktion zwischen dem Frauenanteil im Betrieb einen negativen Effekt auf den Lohn von Frauen. In Beckers Modell würde man stattdessen einen positiven Effekt erwarten - genauso wie für den Frauenanteil im Management. Dagegen hat die RIT keine Probleme diese unterschiedlichen Effekte zu deuten: Die bloße Anzahl an Frauen im Vergleich zu Männern im Betrieb ist keine Ressource. Im Gegenteil: Angesichts des hohen Grads an Institutionalisierung der Kategorie wirkt sich der Frauenanteil sogar negativ auf den Lohn von Frauen aus. Der Frauenanteil im Management kann dagegen als Ressource im claims-making verstanden werden, die den GWG senkt.

14 Statt als Diskriminierungsindikator kann man dieses Merkmal auch als Exklusionsindikator auffassen, wonach Frauen von hoch entlohnenden Betrieben ausgeschlossen werden (Avent-Holt \& Tomaskovic-Devey, 2012). 
Die Interaktion zwischen Geschlecht und der Differenz im Anteil an Hochschulabschlüssen im Betrieb ist ebenfalls signifikant positiv. Der relative Lohn von Frauen steigt also mit zunehmenden Vorteilen beim Anteil an Hochschulabschlüssen im Vergleich zu den männlichen Kollegen im Betrieb. Damit erhalten alle Frauen in solchen Betrieben einen höheren Lohn, in denen diese Statusrelation eher Frauen bevorzugt - unabhängig von individueller Bildung und weiteren Personenmerkmalen. Anders als erwartet, ist der Effekt allerdings nicht schwächer in tarifgebundenen Betrieben, sondern leicht stärker. Wird in Modell 2 zusätzlich für den individuellen beruflichen Rang kontrolliert, wird der Effekt deutlich kleiner. Dies ist ein Hinweis auf Chancenhortung. Die Aufwertung von Frauen als Statusgruppe relativ zu den männlichen Kollegen verbessert die Erfolgschancen im claimsmaking, was sich über die besseren Möglichkeiten des Zugangs zu guten Positionen im Betrieb auf den Lohn auswirkt. In Ostdeutschland ist der Einfluss dieser Statusrelation stärker und geht auch in Modell 2 nur leicht zurück, was darauf hindeutet, dass hier die relativen Löhne von Frauen auch innerhalb von Positionen positiv beeinflusst werden.

Tabelle 1: Multilevel Modelle getrennt nach Tarifbindung, Westdeutschland

\begin{tabular}{|c|c|c|c|c|c|c|}
\hline & \multicolumn{3}{|c|}{ Individuelle Lohnverhandlungen } & \multicolumn{3}{|c|}{ Tarifbindung } \\
\hline & M1 & M2 & M3 & M1 & M2 & M3 \\
\hline Frau * Anteil Frauen im Ma- & $0,212^{* * *}$ & $0,183^{* \star \star}$ & $0,184^{* * *}$ & $0,143^{* * *}$ & $0,122^{* * *}$ & $0,124^{* *}$ \\
\hline nagement & $(0,01)$ & $(0,01)$ & $(0,01)$ & $(0,02)$ & $(0,02)$ & $(0,02)$ \\
\hline Frau * Differenz Anteil an & $0,062^{* *}$ & 0,024 & $-0,012$ & $0,072^{* *}$ & 0,042 & 0,025 \\
\hline Hochschulabschl. & $(0,02)$ & $(0,02)$ & $(0,02)$ & $(0,03)$ & $(0,03)$ & $(0,03)$ \\
\hline \multirow[t]{2}{*}{ Beruflicher Rang } & & $0.525^{\star \star \star}$ & $0.544^{* * *}$ & & $0.474^{* * *}$ & $0.470^{* *}$ \\
\hline & & $(0.01)$ & $(0.01)$ & & $(0.01)$ & $(0.01)$ \\
\hline \multirow[t]{2}{*}{ Frau * berufl. Rang } & & & $-0.060^{* * \star}$ & & & 0.006 \\
\hline & & & $(0.01)$ & & & $(0.01)$ \\
\hline \multirow[t]{2}{*}{ Frau * Bildungsjahre } & & & $-0.005^{* \star \star}$ & & & $-0.009^{* \star}$ \\
\hline & & & $(0.00)$ & & & $(0.00)$ \\
\hline \multirow[t]{2}{*}{ Frau * Jahr im Betrieb } & & & $-0.001^{* *}$ & & & $-0.000^{*}$ \\
\hline & & & $(0.00)$ & & & $(0.00)$ \\
\hline \multirow[t]{2}{*}{ Frau * Befristung } & & & $0.050^{* * *}$ & & & $0.036^{* *}$ \\
\hline & & & $(0.01)$ & & & $(0.01)$ \\
\hline
\end{tabular}

Anmerkung: Standardfehler in Klammern. Alle Modelle beinhalten die genannten Personen-, Betriebs-, und Branchenkontrollvariablen. Die vollständigen Modelle sind im Anhang dargestellt (Tabelle A1). $* \mathrm{p}<0,05 ; * * \mathrm{p}<0,01 ; * * * \mathrm{p}<0,001$.

In Modell 3 werden schließlich weitere Interaktionen zwischen Geschlecht und individuellen Ressourcen wie dem beruflichem Rang, Bildungsjahren, Betriebszugehörigkeitsdauer und Befristung hinzugefügt. Die Interaktionen zeigen die erwarteten negativen Koeffizienten: Der Lohnanstieg pro Bildungsjahr und pro Jahr im Betrieb fällt bei Frauen geringer aus als bei Männern. Lediglich in Befristung scheinen Frauen mehr zu verdienen als Männer. Unterschiede zwischen Betrieben mit und ohne Tarifbindung zeigen sich bei der Interaktion mit beruflichem Rang: Während es in tarifgebundenen Betrieben keinen Effekt gibt, erhalten Frauen in Betrieben ohne Tarifbindung auf dem gleichen beruflichen Rang einen geringeren Lohn als ihre männlichen Kollegen. Hier zeigt sich erneut die Bedeutung betrieblicher claims-making-Prozesse aufgrund der fehlenden überbetrieblichen Standardisierung von Löhnen in Betrieben mit individuellen Lohnverhandlungen. 
In Abschnitt 5.1 wurde gezeigt, dass der GWG entlang der Verteilung größer wird. Die folgenden Ergebnisse der Quantilsregressionen zeigen, inwiefern die in den Multilevel Modellen gefundenen Einflüsse entlang der Lohnverteilung variieren.

\subsection{Der Einfluss betrieblicher Statusrelationen entlang der Verteilung}

Abbildung 3 zeigt die Ergebnisse von mehreren unkonditionalen Quantilsregressionen. Jeder Punkt repräsentiert den Koeffizienten der Interaktion von Frau und Frauenanteil im Management aus einem vollständigen Modell ohne die zusätzlichen Interaktionen (M2), das für insgesamt 21 Quantile berechnet wurde. ${ }^{15}$ Unbedingte Quantilsregressionen lassen sich ähnlich wie OLS-Regressionen interpretieren, die Veränderung im Lohn bezieht sich nur nicht auf den Mittelwert, sondern auf das entsprechende Quantil.

Abbildung 3: Effekt der Interaktion von Frauen mit dem Frauenanteil im Management, Westdeutschland

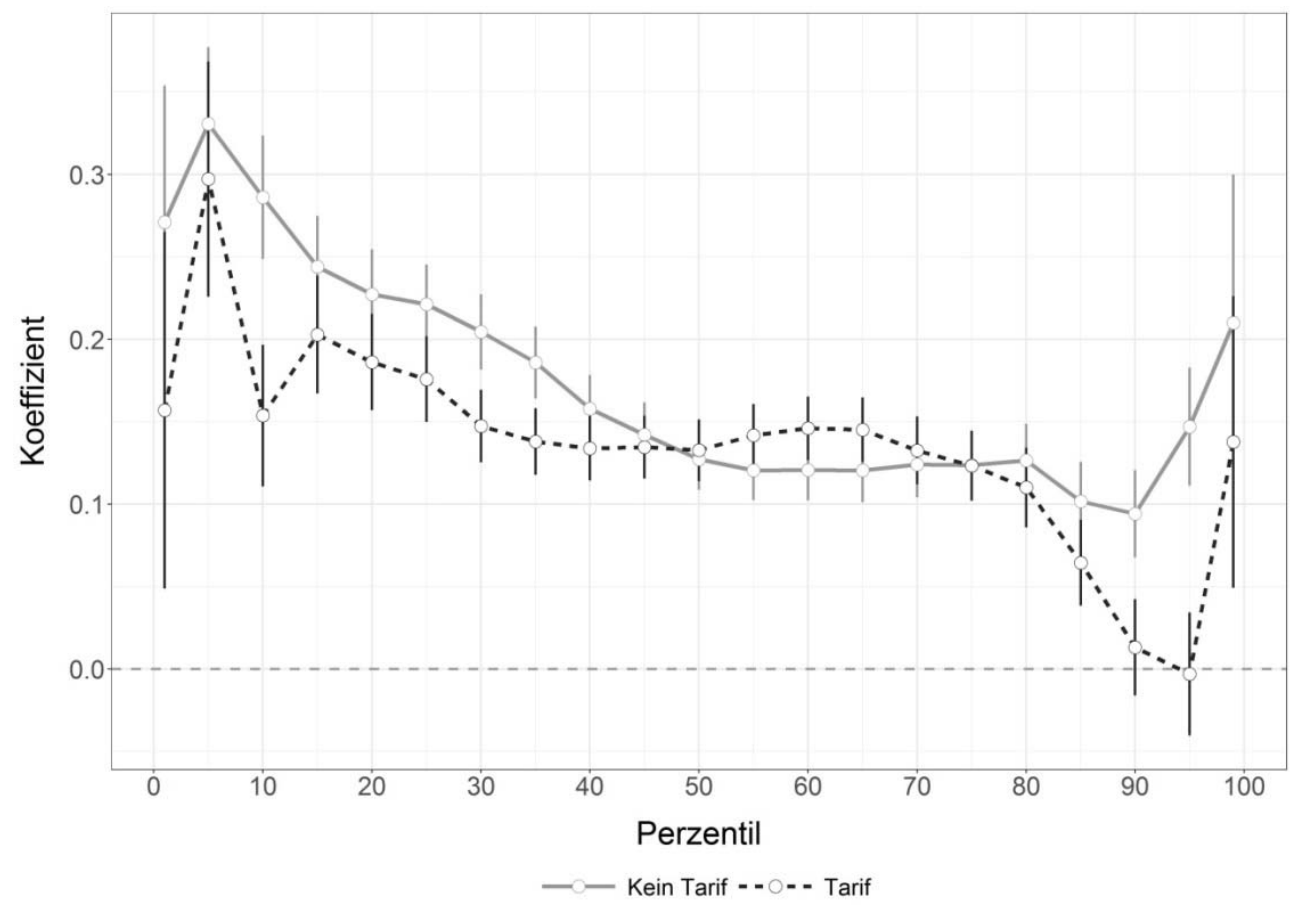

Anmerkung: Die Koeffizienten stammen aus je 21 Quantilsregression, die für jedes der dargestellten Perzentile getrennt nach Tarifbindung geschätzt wurden. Alle Modelle beinhalten die genannten Personen-, Betriebs-, und Branchenkontrollvariablen (M2). Vertikale Linien stellen 95\%-Konfidenzintervalle dar.

15 Die Modelle 1 und 3 ähneln den hier gezeigten Modellen stark. Die Koeffizienten aus diesen Modellen sowie diejenigen für Ostdeutschland werden hier nicht dargestellt, können interessierten Lesern aber auf Anfrage zur Verfügung gestellt werden. 
Abbildung 3 zeigt zunächst, dass die Effekte auf fast alle Perzentile positiv sind. Es ist also irrelevant, ob es sich um hohe oder niedrige Löhne handelt: Frauen jeder Lohngruppe profitieren davon, wenn sie in einem Betrieb arbeiten, in dem der Frauenanteil im Management hoch ist. Darüber hinaus zeigt sich aber, dass, entgegen den Erwartungen, der Effekt für die unteren Lohngruppen stärker ist als für die oberen. Es gibt zwar einen Anstieg der Effekte ab dem 90. Perzentil, der auf die vermuteten Zusammenhänge hindeutet (nämlich die höhere Wirksamkeit von Leistungs- und Kompetenzargumenten bei höheren Löhnen). Die hohen Effekte in der unteren Hälfte der Verteilung könnten aber eher dafür sprechen, dass Betriebe mit einem hohen Frauenanteil im Management größeren Wert auf den Abbau solcher geschlechtsspezifischer Lohnungleichheit legen, was gerade unteren Lohngruppen zugute kommt. Dies ist auch der Lohnbereich, in dem sich Tarifverträge am stärksten positiv auf den Lohn beider Geschlechter auswirken (Fitzenberger, Kohn \& Lembcke, 2013; Kohn \& Lembcke, 2007), weshalb es in tarifgebundenen Betrieben vermutlich weniger Spielraum für die relative Verbesserung der Löhne von Frauen gibt. In Ostdeutschland zeigt sich dagegen der erwartete Anstieg der Effekte über die Verteilung.

Das entgegengesetzte Bild zeigt sich in Abbildung 4. Während steigende Bildungsvorteile von Frauen für untere bis mittlere Lohngruppen (bis zum Median) zu relativen Lohnverlusten führen, wirkt sich diese Statusrelation positiv auf den relativen Lohn der Frauen im oberen Teil der Lohnverteilung aus. Hochschulabschlüsse weisen auf Kompetenz und Leistungsfähigkeit hin. Diese Erwartungen können vor allem Frauen mit hohen Lohnniveaus dazu nutzen, ihre Ansprüche gegenüber männlichen Kollegen durchzusetzen, da bei hohen Gehältern und insbesondere Bonuszahlungen häufig mit individueller Leistung und Verantwortung argumentiert wird. Dies zeigt sich vor allem an den starken Effekten bei den Toplöhnen (90., 95. und 99. Perzentil) in Betrieben ohne Tarifbindung. Auch hier ist wieder zu beachten: Es handelt sich um Effekte betrieblicher Statusrelationen und nicht individueller Bildungstitel. Eine vorteilhafte Statusrelation in Bezug auf Bildung wertet den Status von Frauen im Betrieb auf und hilft insbesondere Frauen im oberen Lohnbereich, ihre Ansprüche, deren Legitimation sich in diesem Lohnbereich oft auf individuelle Leistung stützt, glaubhaft anzumelden.

Frauen im unteren Teil der Lohnverteilung profitieren davon allerdings nicht. Vielmehr verringern sich die relativen Löhne der Frauen in diesem Bereich mit steigenden Bildungsvorteilen - wobei dieser negative Effekt in Ostdeutschland schwächer ausfällt. Eine mögliche Erklärung könnte sein, dass eine günstige Relation bei den Hochschulabschlüssen nur bestimmten Berufsgruppen etwas nützt. Gerade im unteren bis mittleren Lohnbereich finden sich viele Ausbildungsberufe, in denen betriebsweite Bildungsvorteile den Status der dortigen Frauen womöglich nicht heben, sondern eher senken. Diese Sicht wird dadurch unterstützt, dass der negative Effekt in tarifgebundenen Betrieben geringer ausfällt (mit Ausnahme des 1. und 5. Perzentils). Zugangsvoraussetzungen und die Aushandlung der Löhne erfolgt hier zumindest teilweise auf der überbetrieblichen Ebene, weshalb die negativen Folgen des claims-makings eingeschränkt sind. 
Abbildung 4: Effekt der Interaktion von Frauen mit der Differenz im Anteil an Hochschulabschlüssen, Westdeutschland

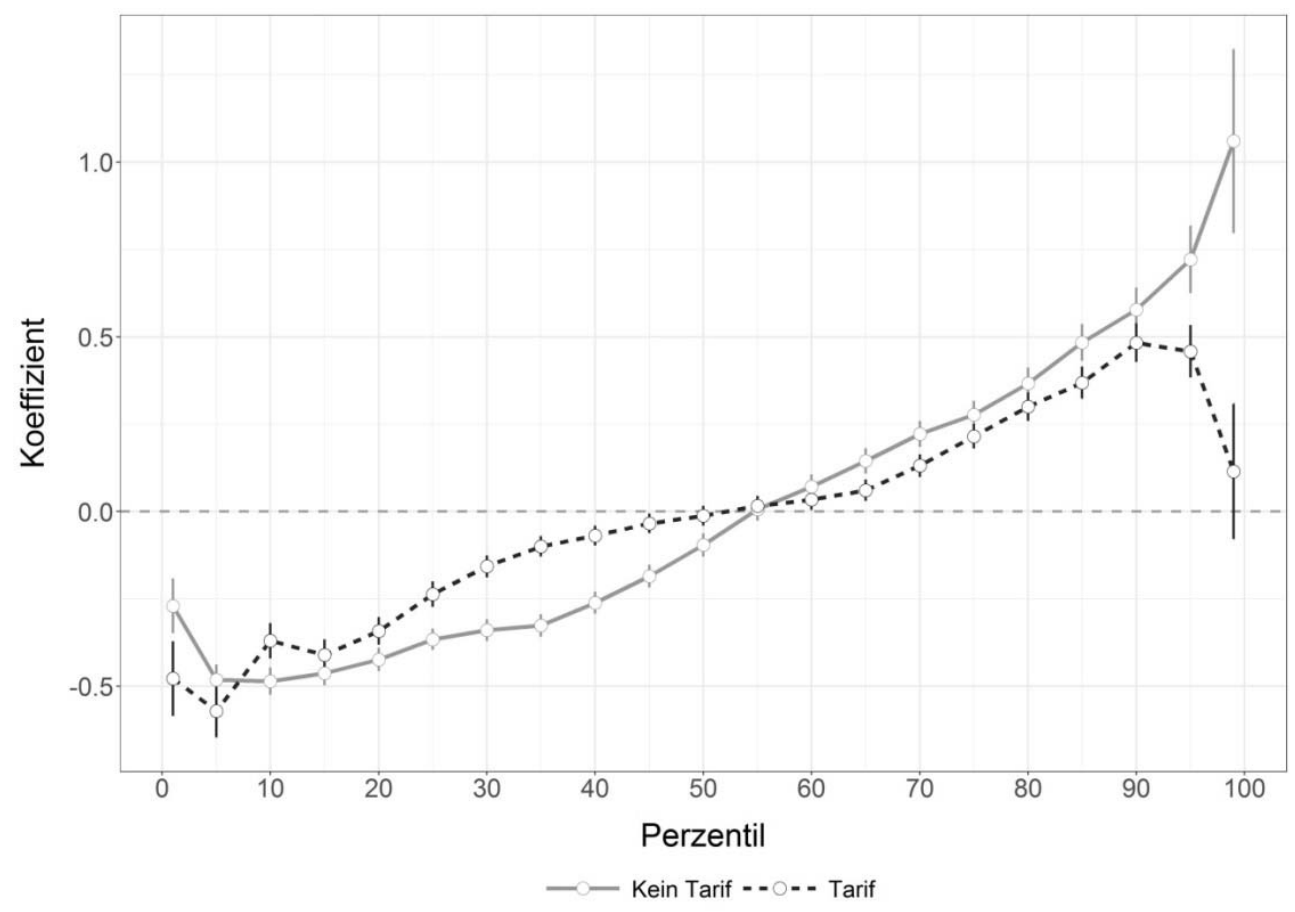

Anmerkung: Die Koeffizienten stammen aus je 21 Quantilsregression, die für jedes der dargestellten Perzentile getrennt nach Tarifbindung geschätzt wurden. Alle Modelle beinhalten die genannten Personen-, Betriebs-, und Branchenkontrollvariablen (M2). Vertikale Linien stellen 95\%-Konfidenzintervalle dar.

Zusammenfassend ergibt sich also ein durchaus differenziertes Bild des Einflusses von betrieblichen Statusrelationen auf die geschlechtsspezifische Lohnungleichheit. Alle Frauen profitieren von einer Beschäftigung in Betrieben mit einem hohen Frauenanteil im Management - jedoch insbesondere Frauen in unteren Lohngruppen in Betrieben ohne Tarifbindung. Wachsende Bildungsvorteile von Frauen im Betrieb erhöhen die relativen Löhne nur im oberen Bereich, während sie für Frauen in unteren Lohngruppen sogar negative Konsequenzen haben.

\section{Diskussion und Fazit}

In der Literatur setzt sich zunehmend die Erkenntnis durch, dass die Arbeitsorganisation ein zentraler Ort für die Herstellung von Lohnungleichheit ist. Mit der Theorie der relationalen Ungleichheit wurde hier ein Modell diskutiert, das die Entstehung von Ungleichheit als interaktionalen Prozess zwischen verschiedenen Akteuren im Betrieb betrachtet. Akteure 
formulieren Lohnansprüche und versuchen diesen Anspruch gegenüber anderen Akteuren durchzusetzen, indem sie relevanten Entscheidern im Betrieb die Legitimität ihres Anspruchs verdeutlichen (claims-making). Entscheidend für den Erfolg ist der Besitz von Statuskategorien in Relation zu anderen Akteuren. Die relativen Löhne von Frauen sollten demnach umso höher ausfallen, je eher betriebliche Statusrelationen zu ihren Gunsten ausfallen und damit die Legitimität von Lohnansprüchen begründen.

Mit den Daten der Verdienststrukturerhebung 2010 und Multilevel Modellen konnte gezeigt werden, dass das Ausmaß des GWG zwischen Betrieben variiert, und zwar als Funktion der betrieblichen Statusrelationen entlang der Geschlechtskategorie: Mit steigendem Anteil von Frauen im Management eines Betriebes und mit steigenden Vorteilen beim Besitz von Hochschulabschlüssen im Vergleich zu Männern erhöht sich der relative Lohn von Frauen. Für letztere Statusrelation konnten zudem Hinweise auf Chancenhortung gefunden werden. Hier übersetzt sich der Vorteil von Frauen im claims-making teilweise in einen leichteren Zugang zu besser bezahlten Positionen im Betrieb.

Mit Quantilsregressionen konnte darüber hinaus dargestellt werden, dass die Interaktionseffekte des Frauenindikators mit dem Frauenanteil im Management und mit den Unterschieden beim Anteil an Hochschulabschlüssen über die Lohnverteilung variieren. Während ein steigender Frauenanteil im Management eines Betriebs vor allem Frauen in unteren Lohngruppen zugutekommt, profitieren Frauen mit hohen Lohnniveaus stärker von Vorteilen bei den Hochschulabschlüssen. Frauen im unteren Bereich der Lohnverteilung zeigen sogar einen negativen Effekt, der impliziert, dass sich der GWG in diesem Teil der Verteilung mit steigenden Bildungsvorteilungen von Frauen im Betrieb vergrößert.

Leider handelt es sich bei der VSE nicht um ein Panel, weshalb die vorliegenden Ergebnisse nicht kausal interpretiert werden können. Auch die Identifikation von Chancenhortungsprozessen würde von solchen Daten profitieren. Dennoch weisen die Ergebnisse auf die große Bedeutung des betrieblichen Kontextes für die Herstellung von Lohnungleichheiten hin und reihen sich damit in einen größer werdenden Forschungszweig ein, der auf diese Zusammenhänge hinweist (Beblo, Ohlert \& Wolf, 2011; Heinze \& Wolf, 2010). Auch nach Kontrolle der typischen Humankapitalfaktoren finden sich signifikante Einflüsse betrieblicher Statusrelationen auf den relativen Lohn von Frauen. Dies stützt einige zentrale Implikationen der RIT, sollte aber gleichzeitig Anstoß für weitere empirische Überprüfungen der Theorie geben. Aus sozialpolitischer Sicht wird die anhaltende Bedeutung von Tarifverträgen für den Abbau von Lohnungleichheiten zwischen den Geschlechtern unterstrichen. Ferner liefern diese Ergebnisse weitere Argumente für den Nutzen eines stärker egalitär besetzten Managements für die Reduzierung geschlechtsspezifischer Lohnungleichheit.

\section{Literaturverzeichnis}

Acker, J. (1990). Hierarchies, jobs, bodies: A theory of gendered organizations. Gender \& Society, 4 (2), 139-158. https://doi.org/10.1177/089124390004002002

Arulampalam, W., Booth, A. L., \& Bryan, M. L. (2007). Is there a glass ceiling over Europe? Exploring the gender pay gap across the wage distribution. Industrial and Labor Relations Review, 60 (2), 163-186. https://doi.org/10.2307/25249069

Avent-Holt, D. \& Tomaskovic-Devey, D. (2012). Relational Inequality: Gender Earnings Inequality in U.S. and Japanese Manufacturing Plants in the Early 1980s. Social Forces, 91 (1), 157-180. https://doi.org/10.1093/sf/sos068 
Avent-Holt, D. \& Tomaskovic-Devey, D. (2014). A relational theory of earnings inequality. American Behavioral Scientist, 58 (3), 379-399. https://doi.org/10.1177/0002764213503337

Baron, J. N. (1984). Organizational perspectives on stratification. Annual Review of Sociology, 10, 37-69. Verfügbar unter http://www.jstor.org/stable/2083167

Baron, J. N. \& Bielby, W. T. (1980). Bringing the firms back in: Stratification, segmentation, and the organisation of work. American Sociological Review, 45, 737-765.

Beblo, M., Ohlert, C. \& Wolf, E. (2011). Logib-D und die Entgeltunterschiede zwischen Männern und Frauen in deutschen Betrieben - Eine Abschätzung des politischen Handlungsfeldes. Zeitschrift für ArbeitsmarktForschung, 44 (1-2), 43-52. https://doi.org/10.1007/s12651-011-0075-5

Becker, G. S. (1971). The economics of discrimination (Economics research studies of the Economics Research Center of the University of Chicago, 2d ed). Chicago: University of Chicago Press.

Blau, F. D. \& Kahn, L. M. (2016). The gender wage gap: Extent, trends, and explanations. National Bureau of Economic Research Working Paper Series, No. 21913. https://doi.org/10.3386/w21913

Broadbridge, A. \& Hearn, J. (2008). Gender and management. New directions in research and continuing patterns in practice. British Journal of Management, 19 (1), 38-49. https://doi.org/10.1111/j.1467-8551.2008.00570.x

Cardoso, A. R., Guimarães, P. \& Portugal, P. (2016). What drives the gender wage gap? A look at the role of firm and job-title heterogeneity. Oxford Economic Papers, 68 (2), 506-524. https://doi.org/10.1093/oep/gpv069

Cardoso, A. R. \& Winter-Ebmer, R. (2010). Female-led firms and gender wage policies. Industrial and Labor Relations Review, 64 (1), 143-163.

Castilla, E. J. (2011). Bringing Managers Back In: Managerial Influences on Workplace Inequality. American Sociological Review, 76 (5), 667-694. https://doi.org/10.1177/0003122411420814

Cohen, P. N. \& Huffman, M. L. (2007). Working for the woman? Female managers and the gender wage gap. American Sociological Review, 72 (5), 681-704. https://doi.org/10.1177/000312240707200502

Diewald, M. \& Faist, T. (2011). Von Heterogenitäten zu Ungleichheiten: Soziale Mechanismen als Erklärungsansatz der Genese sozialer Ungleichheiten. Berliner Journal für Soziologie, 21 (1), 91114. https://doi.org/10.1007/s11609-011-0144-1

Firpo, S., Fortin, N. \& Lemieux, T. (2009). Unconditional quantile regressions. Econometrica, 77 (3), 953-973. https://doi.org/10.3982/ECTA6822

Fitzenberger, B., Kohn, K. \& Lembcke, A. C. (2013). Union density and varieties of coverage: The anatomy of union wage effects in Germany. Industrial and Labor Relations Review, 66 (1), 169197.

Gartner, H. \& Hinz, T. (2009). Geschlechtsspezifische Lohnungleichheit in Betrieben, Berufen und Jobzellen (1993-2006). Berliner Journal für Soziologie, 19 (4), 557-575. https://doi.org/10.1007/s11609-009-0110-3

Gorman, E. H. (2005). Gender stereotypes, same-gender preferences, and organizational variation in the hiring of women: Evidence from law firms. American Sociological Review, 70 (4), 702-728. https://doi.org/10.1177/000312240507000408

Günther, R. (2013). Methodik der Verdienststrukturerhebung 2010. Wirtschaft und Statistik, 2, 127-142.

Heinze, A. 2009. Earnings of men and women in firms with a female dominated workforce: What drives the impact of sex segregation on wages? (ZEW Discussion Paper 09-012). Verfügbar unter http://ideas.repec.org/p/zbw/zewdip/7537.html

Heinze, A. \& Wolf, E. (2010). The intra-firm gender wage gap: a new view on wage differentials based on linked employer-employee data. Journal of Population Economics, 23 (3), 851-879. https://doi.org/10.1007/s00148-008-0229-0 
Hensvik, L. E. (2014). Manager impartiality. Worker-firm matching and the gender wage gap. Industrial and Labor Relations Review, 67 (2), 395-421. https://doi.org/10.1177/001979391406700205

Hirsch, B. (2013). The impact of female managers on the gender pay gap. Evidence from linked employer-employee data for Germany. Economics Letters, 119 (3), 348-350. https://doi.org/10.1016/j.econlet.2013.03.021

Holgersson, C. (2013). Recruiting managing directors. Doing homosociality. Gender, Work \& Organization, 20 (4), 454-466. https://doi.org/10.1111/j.1468-0432.2012.00595.x

Huffman, M. L., Cohen, P. N. \& Pearlman, J. (2010). Engendering change. Organizational dynamics and workplace gender desegregation, 1975-2005. Administrative Science Quarterly, 55 (2), 255-277. https://doi.org/10.2189/asqu.2010.55.2.255

Hultin, M. \& Szulkin, R. (1999). Wages and unequal access to organizational power: An empirical test of gender discrimination. Administrative Science Quarterly, 44 (3), 453-472. https://doi.org/10.2307/2666958

Hultin, M. \& Szulkin, R. (2003). Mechanisms of inequality. Unequal access to organizational power and the gender wage gap. European Sociological Review, 19 (2), 143-159. https://doi.org/10.1093/esr/19.2.143

Kassenboehmer, S. C. \& Sinning, M. G. (2014). Distributional changes in the gender wage gap. Industrial and Labor Relations Review, 67 (2), 335-361. https://doi.org/10.1177/001979391406700203

Kohn, K. \& Lembcke, A. C. (2007). Wage distributions by bargaining regime. Wirtschafts- und Sozialstatistisches Archiv, 1 (3-4), 247-261. https://doi.org/10.1007/s11943-007-0023-6

Kunze, A. \& Miller, A. R. (2014). Women helping women? Evidence from private sector data on workplace hierarchies. NBER Working Paper Series, No. 20761. https://doi.org/10.3386/w20761

Kurtulus, F. A. \& Tomaskovic-Devey, D. (2012). Do female top managers help women to advance? A panel study using EEO-1 records. The Annals of the American Academy of Political and Social Science, 639 (1), 173-197. https://doi.org/10.1177/0002716211418445

Lemieux, T., MacLeod, W. B. \& Parent, D. (2009). Performance pay and wage inequality. Quarterly Journal of Economics, 124 (1), 1-49. https://doi.org/10.1162/qjec.2009.124.1.1

Lips, H. M. (2013). The gender pay gap. Challenging the rationalizations. Perceived equity, discrimination, and the limits of human capital models. Sex Roles, 68 (3-4), 169-185. https://doi.org/10.1007/s11199-012-0165-z

Ludsteck, J. (2014). The impact of segregation and sorting on the gender wage gap. Evidence from German linked longitudinal employer-employee data. Industrial and Labour Review, 67 (2), 362394.

Moss, P. I. \& Tilly, C. (2003). Stories employers tell. Race, skill, and hiring in America (A volume in the multi-city study of urban inequality). New York: Russell Sage Foundation.

Nelson, R. L. \& Bridges, W. P. (1999). Legalizing gender inequality. Courts, markets, and unequal pay for women in America (Structural analysis in the social sciences, vol. 16). Cambridge [England]: Cambridge University Press.

Projektgruppe GiB. (2010). Geschlechterungleichheiten im Betrieb. Arbeit, Entlohnung und Gleichstellung in der Privatwirtschaft (Forschung aus der Hans-Böckler-Stiftung, Bd. 110, 1. Aufl.). Berlin: Edition sigma.

Rabe-Hesketh, S. \& Skrondal, A. (2012). Multilevel and longitudinal modeling using stata (A Stata Press publication, Third edition). College Station, Texas: StataCorp LP.

Ridgeway, C. L. (1997). Interaction and the conservation of gender inequality: Considering employment. American Sociological Review, 62 (2), 218-235. https://doi.org/10.2307/2657301 
Roscigno, V. J., Garcia, L. M. \& Bobbitt-Zeher, D. (2007). Social closure and processes of race/sex employment discrimination. The Annals of the American Academy of Political and Social Science, 609 (1), 16-48. https://doi.org/10.1177/0002716206294898

Schweiker, M. \& Groß, M. (2017). Organizational environments and bonus payments. Rent destruction or rent sharing? Research in Social Stratification and Mobility, 37, 7-19. https://doi.org/10.1016/j.rssm.2016.04.005

Sommerfeld, K. (2013). Wage inequality and employment in the German labor market. Freiburg im Breisgau: Universität Freiburg.

Stainback, K., Tomaskovic-Devey, D. \& Skaggs, S. (2010). Organizational approaches to inequality: Inertia, relative power, and environments. Annual Review of Sociology, 36 (1), 225-247. https://doi.org/10.1146/annurev-soc-070308-120014

Statistisches Bundesamt. (2016). Verdienstunterschied zwischen Frauen und Männern in Deutschland bei $21 \%$. Wiesbaden. Verfügbar unter

https://www.destatis.de/DE/PresseService/Presse/Pressemitteilungen/2016/03/PD16_097_621.html

Tilly, C. (1998). Durable inequality. Berkeley: University of California Press.

Tomaskovic-Devey, D. (2014). The relational generation of workplace inequalities. Social Currents, 1 (1), 51-73. https://doi.org/10.1177/2329496513514032

Tomaskovic-Devey, D., Hällsten, M. \& Avent-Holt, D. (2015). Where do immigrants fare worse? Modeling workplace wage gap variation with longitudinal employer-employee data. American Journal of Sociology, 120 (4), 1095-1143. https://doi.org/10.1086/679191

\section{Anhang}

Tabelle A1: Vollständige Multilevel Modelle, Westdeutschland

\begin{tabular}{|c|c|c|c|c|c|c|}
\hline & \multicolumn{3}{|c|}{ Individuelle Lohnverhandlungen } & \multicolumn{3}{|c|}{ Tarifbindung } \\
\hline & M1 & $\mathrm{M} 2$ & M3 & M1 & M2 & M3 \\
\hline \multirow[t]{2}{*}{ Frau } & $-0.144^{* \star \star}$ & $-0.162^{* * \star}$ & $-0.058^{* \star \star}$ & $-0.107^{\star \star \star}$ & $-0.112^{* * *}$ & 0.005 \\
\hline & $(0.01)$ & $(0.01)$ & $(0.02)$ & $(0.01)$ & $(0.01)$ & $(0.01)$ \\
\hline \multirow[t]{2}{*}{ Teilzeit } & $-0.066^{\star \star \star}$ & $-0.053^{* \star *}$ & $-0.053^{* \star *}$ & $-0.057^{* * *}$ & $-0.046^{* * *}$ & $-0.046^{* * *}$ \\
\hline & $(0.00)$ & $(0.00)$ & $(0.00)$ & $(0.00)$ & $(0.00)$ & $(0.00)$ \\
\hline \multirow[t]{2}{*}{ Bildungjahre } & $0.067^{\star \star \star}$ & $0.045^{* * *}$ & $0.046^{\star \star \star}$ & $0.069^{* * *}$ & $0.046^{* \star *}$ & $0.050^{* *}$ \\
\hline & $(0.00)$ & $(0.00)$ & $(0.00)$ & $(0.00)$ & $(0.00)$ & $(0.00)$ \\
\hline \multirow[t]{2}{*}{ Jahre im Betrieb } & $0.006^{\star \star \star}$ & $0.006^{* *}$ & $0.006^{* \star \star}$ & $0.005^{* \star *}$ & $0.004^{* * *}$ & $0.004^{* * *}$ \\
\hline & $(0.00)$ & $(0.00)$ & $(0.00)$ & $(0.00)$ & $(0.00)$ & $(0.00)$ \\
\hline \multirow[t]{2}{*}{ Alter in Jahren } & $0.043^{\star \star *}$ & $0.042^{\star \star \star}$ & $0.042^{* * *}$ & $0.034^{* * *}$ & $0.034^{* * *}$ & $0.034^{* * *}$ \\
\hline & $(0.00)$ & $(0.00)$ & $(0.00)$ & $(0.00)$ & $(0.00)$ & $(0.00)$ \\
\hline \multirow[t]{2}{*}{ Alter in Jahren (quad.) } & $-0.000^{\star * \star}$ & $-0.000^{* * *}$ & $-0.000^{* \star *}$ & $-0.000^{* * *}$ & $-0.000^{* * *}$ & $-0.000^{* * *}$ \\
\hline & $(0.00)$ & $(0.00)$ & $(0.00)$ & $(0.00)$ & $(0.00)$ & $(0.00)$ \\
\hline \multirow[t]{2}{*}{ Befristung } & $-0.156^{\star \star \star}$ & $-0.141^{\star \star \star}$ & $-0.167^{\star \star \star}$ & $-0.117^{\star \star \star}$ & $-0.122^{* * *}$ & $-0.138^{* * *}$ \\
\hline & $(0.01)$ & $(0.01)$ & $(0.01)$ & $(0.01)$ & $(0.01)$ & $(0.01)$ \\
\hline \multirow[t]{2}{*}{ \%Frauen im Mana. } & $-0.134^{\star \star \star}$ & $-0.110^{\star * *}$ & $-0.108^{\star \star \star}$ & $-0.096^{* * *}$ & $-0.075^{* * *}$ & $-0.076^{* * *}$ \\
\hline & $(0.02)$ & $(0.02)$ & $(0.02)$ & $(0.02)$ & $(0.02)$ & $(0.02)$ \\
\hline \multirow[t]{2}{*}{ Diff. \%Hochschul. } & $-0.180^{* * *}$ & $-0.112^{\star * *}$ & $-0.083^{* *}$ & $-0.190^{* * *}$ & $-0.139^{* * *}$ & $-0.118^{* * *}$ \\
\hline & $(0.03)$ & $(0.03)$ & $(0.03)$ & $(0.04)$ & $(0.03)$ & $(0.03)$ \\
\hline \multirow[t]{2}{*}{$\varnothing$ Bildungsjahre im Betr. } & $0.048^{\star \star \star}$ & $0.034^{\star \star \star}$ & $0.037^{\star \star \star}$ & $0.058^{* \star \star}$ & $0.041^{* \star *}$ & $0.044^{* * *}$ \\
\hline & $(0.00)$ & $(0.00)$ & $(0.00)$ & $(0.00)$ & $(0.00)$ & $(0.00)$ \\
\hline$\varnothing$ Jahre im Betrieb & $0.009^{\star \star \star}$ & $0.007^{\star \star \star}$ & $0.008^{\star \star \star}$ & $0.007^{* \star \star}$ & $0.005^{* * *}$ & $0.005^{* * *}$ \\
\hline
\end{tabular}




\begin{tabular}{|c|c|c|c|c|c|c|}
\hline & \multicolumn{3}{|c|}{ Individuelle Lohnverhandlungen } & \multicolumn{3}{|c|}{ Tarifbindung } \\
\hline & M1 & $\mathrm{M} 2$ & M3 & M1 & M2 & M3 \\
\hline & $(0.00)$ & $(0.00)$ & $(0.00)$ & $(0.00)$ & $(0.00)$ & $(0.00)$ \\
\hline \multirow[t]{2}{*}{ \%Frauen im Betrieb } & $-0.068^{* *}$ & -0.022 & -0.013 & -0.044 & 0.017 & 0.014 \\
\hline & $(0.03)$ & $(0.02)$ & $(0.02)$ & $(0.04)$ & $(0.03)$ & $(0.03)$ \\
\hline \multirow[t]{2}{*}{ Betriebsgröße } & $0.000^{\star \star \star}$ & $0.000^{\star * \star}$ & $0.000^{\star \star \star}$ & $0.000^{* * *}$ & $0.000^{* * *}$ & $0.000^{* * *}$ \\
\hline & $(0.00)$ & $(0.00)$ & $(0.00)$ & $(0.00)$ & $(0.00)$ & $(0.00)$ \\
\hline \multirow[t]{2}{*}{ Frau*\%Frauen im Mana. } & $0.212^{\star \star \star}$ & $0.183^{\star \star \star}$ & $0.184^{* \star *}$ & $0.143^{* * *}$ & $0.122^{* * *}$ & $0.124^{* * *}$ \\
\hline & $(0.01)$ & $(0.01)$ & $(0.01)$ & $(0.02)$ & $(0.02)$ & $(0.02)$ \\
\hline \multirow[t]{2}{*}{ Frau*Diff. \%Hochschul. } & $0.062^{* *}$ & 0.024 & -0.012 & $0.072^{* *}$ & 0.042 & 0.025 \\
\hline & $(0.02)$ & $(0.02)$ & $(0.02)$ & $(0.03)$ & $(0.03)$ & $(0.03)$ \\
\hline \multirow[t]{2}{*}{ Frau*\%Frauen im Betr. } & $-0.160^{* \star *}$ & $-0.094^{* * *}$ & $-0.117^{* \star *}$ & $-0.102^{* * *}$ & $-0.069^{* * *}$ & $-0.068^{* * *}$ \\
\hline & $(0.02)$ & $(0.02)$ & $(0.02)$ & $(0.02)$ & $(0.02)$ & $(0.02)$ \\
\hline Baugewerbe (Ref.) & I & I & l & l & I & / \\
\hline \multirow[t]{2}{*}{ Bergbau } & $0.205^{* *}$ & $0.218^{* *}$ & $0.214^{* *}$ & 0.024 & 0.020 & 0.020 \\
\hline & $(0.07)$ & $(0.07)$ & $(0.07)$ & $(0.07)$ & $(0.06)$ & $(0.06)$ \\
\hline \multirow[t]{2}{*}{ Verarbeitendes Gewerbe } & $0.098^{*}$ & $0.096^{*}$ & $0.092^{*}$ & $0.150^{* * *}$ & $0.128^{* * *}$ & $0.128^{* * *}$ \\
\hline & $(0.05)$ & $(0.05)$ & $(0.05)$ & $(0.04)$ & $(0.04)$ & $(0.04)$ \\
\hline \multirow[t]{2}{*}{ Energie- und Wasserversorgung } & $0.345^{\star \star \star}$ & $0.330^{\star * \star}$ & $0.327^{\star \star \star}$ & $0.118^{* *}$ & $0.105^{* *}$ & $0.105^{* *}$ \\
\hline & $(0.05)$ & $(0.05)$ & $(0.05)$ & $(0.05)$ & $(0.04)$ & $(0.04)$ \\
\hline \multirow[t]{2}{*}{ Handel } & $0.125^{*}$ & $0.116^{*}$ & $0.114^{*}$ & 0.045 & 0.045 & 0.045 \\
\hline & $(0.05)$ & $(0.05)$ & $(0.05)$ & $(0.05)$ & $(0.04)$ & $(0.04)$ \\
\hline \multirow[t]{2}{*}{ Kredit- und Versicherungsgewerbe } & $0.390^{\star \star *}$ & $0.304^{* * *}$ & $0.306^{* * *}$ & $0.151^{* *}$ & 0.012 & 0.012 \\
\hline & $(0.05)$ & $(0.05)$ & $(0.05)$ & $(0.05)$ & $(0.04)$ & $(0.04)$ \\
\hline \multirow[t]{2}{*}{ Gastgewerbe } & 0.008 & $0.129^{*}$ & $0.119^{*}$ & $-0.135^{*}$ & -0.049 & -0.050 \\
\hline & $(0.05)$ & $(0.05)$ & $(0.05)$ & $(0.05)$ & $(0.05)$ & $(0.05)$ \\
\hline \multirow[t]{2}{*}{ Verkehr und Lagerei } & $0.138^{* *}$ & $0.118^{*}$ & $0.115^{*}$ & 0.037 & 0.023 & 0.023 \\
\hline & $(0.05)$ & $(0.05)$ & $(0.05)$ & $(0.05)$ & $(0.04)$ & $(0.04)$ \\
\hline \multirow[t]{2}{*}{ Grundstück- und Wohnungswesen } & $0.129^{* *}$ & $0.107^{*}$ & $0.104^{*}$ & -0.039 & -0.053 & -0.054 \\
\hline & $(0.05)$ & $(0.05)$ & $(0.05)$ & $(0.04)$ & $(0.04)$ & $(0.04)$ \\
\hline \multirow[t]{2}{*}{ Erziehung und Unterricht } & 0.027 & 0.012 & 0.008 & -0.051 & -0.049 & -0.048 \\
\hline & $(0.06)$ & $(0.06)$ & $(0.06)$ & $(0.06)$ & $(0.05)$ & $(0.05)$ \\
\hline \multirow[t]{2}{*}{ Gesundheits- und Sozialwesen } & $0.155^{* *}$ & $0.188^{* * *}$ & $0.181^{* * *}$ & 0.036 & 0.048 & 0.049 \\
\hline & $(0.05)$ & $(0.05)$ & $(0.05)$ & $(0.05)$ & $(0.04)$ & $(0.04)$ \\
\hline \multirow[t]{2}{*}{ Sons. Dienstleistungen } & $0.102^{*}$ & $0.098^{*}$ & $0.095^{*}$ & -0.048 & -0.048 & -0.048 \\
\hline & $(0.05)$ & $(0.05)$ & $(0.05)$ & $(0.05)$ & $(0.04)$ & $(0.04)$ \\
\hline \multirow[t]{2}{*}{ Beruflicher Rang } & & $0.525^{* * *}$ & $0.544^{* * *}$ & & $0.474^{* * *}$ & $0.470^{\ldots+*}$ \\
\hline & & $(0.01)$ & $(0.01)$ & & $(0.01)$ & $(0.01)$ \\
\hline \multirow[t]{2}{*}{ Frau * berufl. Rang } & & & $-0.060^{* * *}$ & & & 0.006 \\
\hline & & & $(0.01)$ & & & $(0.01)$ \\
\hline \multirow[t]{2}{*}{ Frau * Bildungsjahre } & & & $-0.005^{* \star \star}$ & & & $-0.009^{* * *}$ \\
\hline & & & $(0.00)$ & & & $(0.00)$ \\
\hline \multirow[t]{2}{*}{ Frau * Jahre im Betrieb } & & & $-0.001^{\star *}$ & & & $-0.000^{*}$ \\
\hline & & & $(0.00)$ & & & $(0.00)$ \\
\hline Frau * Befristung & & & $0.050^{* * *}$ & & & $0.036^{* * *}$ \\
\hline & & & $(0.01)$ & & & $(0.01)$ \\
\hline Konstante & $0.299^{\star \star \star}$ & $0.512^{\star * *}$ & $0.438^{\star \star \star}$ & $0.484^{* * *}$ & $0.765^{* * \star}$ & $0.694^{t * *+}$ \\
\hline & $(0.07)$ & $(0.06)$ & $(0.07)$ & $(0.07)$ & $(0.06)$ & $(0.06)$ \\
\hline
\end{tabular}




\begin{tabular}{lllllll}
\hline & \multicolumn{2}{c}{ Individuelle Lohnverhandlungen } & \multicolumn{3}{c}{ Tarifbindung } \\
\hline & \multicolumn{1}{c}{$\mathrm{M} 1$} & $\mathrm{M} 2$ & $\mathrm{M} 3$ & $\mathrm{M} 1$ & $\mathrm{M} 2$ & $\mathrm{M} 3$ \\
\hline $\mathrm{sd}(\mathrm{Frau})$ & $0.126^{* * *}$ & $0.119^{* * *}$ & $0.118^{* * *}$ & $0.094^{* * *}$ & $0.095^{* * *}$ & $0.094^{* * *}$ \\
& $(0.00)$ & $(0.00)$ & $(0.00)$ & $(0.00)$ & $(0.00)$ & $(0.00)$ \\
sd(Konstante) & $0.237^{* * *}$ & $0.214^{* * *}$ & $0.213^{* * *}$ & $0.179^{* * *}$ & $0.160^{* * *}$ & $0.160^{* * *}$ \\
& $(0.00)$ & $(0.02)$ & $(0.00)$ & $(0.00)$ & $(0.00)$ & $(0.00)$ \\
Korrelation(Frau, Konstante) & $-0.610^{* * *}$ & $-0.530^{* * *}$ & $-0.524^{* * *}$ & $-0.518^{* * *}$ & $-0.444^{* * *}$ & $-0.438^{* * *}$ \\
& $(0.02)$ & $(0.02)$ & $(0.02)$ & $(0.00)$ & $(0.04)$ & $(0.04)$ \\
sd(Residuen) & $0.287^{* * *}$ & $0.269^{* * *}$ & $0.269^{* * *}$ & $0.237^{* * *}$ & $0.221^{* * *}$ & $0.221^{* * *}$ \\
& $(0.00)$ & $(0.00)$ & $(0.00)$ & $(0.00)$ & $(0.01)$ & $(0.01)$ \\
\hline & 361.693 & 361.693 & 361.693 & 245.417 & 245.417 & 245.417 \\
\hline
\end{tabular}

${ }^{*} p<0.05,{ }^{* *} p<0.01,{ }^{* * * *} p<0.001$

Tabelle A2: Vollständige Multilevel Modelle, Ostdeutschland

\begin{tabular}{|c|c|c|c|c|c|c|}
\hline & \multicolumn{3}{|c|}{ Individuelle Lohnverhandlungen } & \multicolumn{3}{|c|}{ Tarifbindung } \\
\hline & M1 & M2 & M3 & M1 & M2 & M3 \\
\hline \multirow[t]{2}{*}{$\overline{\text { Frau }}$} & $-0.135^{\star \star \star}$ & $-0.164^{* \star \star}$ & -0.012 & $-0.067^{* \star \star}$ & $-0.092^{\star \star *}$ & $-0.077^{*}$ \\
\hline & $(0.01)$ & $(0.01)$ & $(0.03)$ & $(0.02)$ & $(0.02)$ & $(0.03)$ \\
\hline \multirow[t]{2}{*}{ Teilzeit } & $-0.060^{\star \star \star}$ & $-0.044^{* \star *}$ & $-0.046^{\star \star \star}$ & $-0.069^{* \star \star}$ & $-0.044^{* \star *}$ & $-0.042^{\star \star \star}$ \\
\hline & $(0.01)$ & $(0.01)$ & $(0.01)$ & $(0.01)$ & $(0.01)$ & $(0.01)$ \\
\hline \multirow[t]{2}{*}{ Bildungjahre } & $0.084^{* \star *}$ & $0.054^{* * *}$ & $0.058^{* * *}$ & $0.080^{* * *}$ & $0.051^{\star \star *}$ & $0.053^{* \star *}$ \\
\hline & $(0.00)$ & $(0.00)$ & $(0.00)$ & $(0.00)$ & $(0.00)$ & $(0.00)$ \\
\hline \multirow[t]{2}{*}{ Jahre im Betrieb } & $0.007^{\star \star \star}$ & $0.006^{* \star *}$ & $0.006^{* \star \star}$ & $0.004^{* * *}$ & $0.004^{* * *}$ & $0.003^{* * *}$ \\
\hline & $(0.00)$ & $(0.00)$ & $(0.00)$ & $(0.00)$ & $(0.00)$ & $(0.00)$ \\
\hline \multirow[t]{2}{*}{ Alter in Jahren } & $0.027^{\star \star \star}$ & $0.025^{* \star *}$ & $0.025^{* \star *}$ & $0.034^{* * *}$ & $0.032^{\star * *}$ & $0.032^{* * *}$ \\
\hline & $(0.00)$ & $(0.00)$ & $(0.00)$ & $(0.00)$ & $(0.00)$ & $(0.00)$ \\
\hline \multirow[t]{2}{*}{ Alter in Jahren (quad.) } & $-0.000^{* \star \star}$ & $-0.000^{* * *}$ & $-0.000^{* * *}$ & $-0.000^{* \star \star}$ & $-0.000^{* * *}$ & $-0.000^{* * *}$ \\
\hline & $(0.00)$ & $(0.00)$ & $(0.00)$ & $(0.00)$ & $(0.00)$ & $(0.00)$ \\
\hline \multirow[t]{2}{*}{ Befristung } & $-0.153^{\star \star \star}$ & $-0.138^{* * *}$ & $-0.153^{* \star *}$ & $-0.147^{* \star *}$ & $-0.141^{\star \star * *}$ & $-0.176^{* * *}$ \\
\hline & $(0.01)$ & $(0.01)$ & $(0.01)$ & $(0.01)$ & $(0.01)$ & $(0.02)$ \\
\hline \multirow[t]{2}{*}{ \%Frauen im Mana. } & $-0.145^{\star \star \star}$ & $-0.114^{* * *}$ & $-0.112^{* * *}$ & $-0.100^{*}$ & $-0.090^{+}$ & $-0.089^{\star}$ \\
\hline & $(0.02)$ & $(0.02)$ & $(0.02)$ & $(0.05)$ & $(0.04)$ & $(0.04)$ \\
\hline \multirow[t]{2}{*}{ Diff. \%Hochschul. } & $-0.148^{* *}$ & -0.065 & -0.036 & $-0.257^{* \star \star}$ & $-0.181^{* *}$ & $-0.181^{\star}$ \\
\hline & $(0.05)$ & $(0.05)$ & $(0.05)$ & $(0.08)$ & $(0.07)$ & $(0.07)$ \\
\hline \multirow[t]{2}{*}{$\varnothing$ Bildungsjahre im Betr. } & $0.046^{\star \star \star}$ & $0.028^{* * *}$ & $0.027^{* * \star}$ & $0.037^{* * *}$ & $0.023^{* *}$ & $0.023^{* *}$ \\
\hline & $(0.01)$ & $(0.01)$ & $(0.01)$ & $(0.01)$ & $(0.01)$ & $(0.01)$ \\
\hline \multirow[t]{2}{*}{$\varnothing$ Jahre im Betrieb } & $0.011^{* \star *}$ & $0.010^{* * *}$ & $0.010^{* * *}$ & $0.013^{* \star *}$ & $0.011^{* * *}$ & $0.011^{\star \star *}$ \\
\hline & $(0.00)$ & $(0.00)$ & $(0.00)$ & $(0.00)$ & $(0.00)$ & $(0.00)$ \\
\hline \multirow[t]{2}{*}{ \%Frauen im Betrieb } & 0.011 & 0.045 & 0.046 & -0.068 & -0.060 & -0.063 \\
\hline & $(0.04)$ & $(0.04)$ & $(0.04)$ & $(0.08)$ & $(0.06)$ & $(0.07)$ \\
\hline \multirow[t]{2}{*}{ Betriebsgröße } & $0.000^{\star \star \star *}$ & $0.000^{* * *}$ & $0.000^{* * *}$ & $0.000^{*}$ & $0.000^{* *}$ & $0.000^{* *}$ \\
\hline & $(0.00)$ & $(0.00)$ & $(0.00)$ & $(0.00)$ & $(0.00)$ & $(0.00)$ \\
\hline \multirow[t]{2}{*}{ Frau*\%Frauen im Mana. } & $0.148^{* \star *}$ & $0.100^{* * *}$ & $0.099^{* * *}$ & $0.139^{* * *}$ & $0.121^{\star \star \star *}$ & $0.120^{* * *}$ \\
\hline & $(0.02)$ & $(0.02)$ & $(0.02)$ & $(0.03)$ & $(0.02)$ & $(0.02)$ \\
\hline \multirow[t]{2}{*}{ Frau*Diff. \%Hochschul. } & 0.053 & 0.032 & -0.006 & $0.103^{*}$ & $0.093^{*}$ & $0.095^{*}$ \\
\hline & $(0.03)$ & $(0.03)$ & $(0.03)$ & $(0.05)$ & $(0.04)$ & $(0.05)$ \\
\hline \multirow[t]{2}{*}{ Frau*\%Frauen im Betr. } & $-0.069^{*}$ & 0.023 & 0.004 & $-0.158^{* * *}$ & $-0.091^{*}$ & $-0.086^{*}$ \\
\hline & $(0.03)$ & $(0.03)$ & $(0.03)$ & $(0.04)$ & $(0.04)$ & $(0.04)$ \\
\hline Baugewerbe (Ref.) & / & / & / & / & / & / \\
\hline
\end{tabular}




\begin{tabular}{|c|c|c|c|c|c|c|}
\hline & \multicolumn{3}{|c|}{ Individuelle Lohnverhandlungen } & \multicolumn{3}{|c|}{ Tarifbindung } \\
\hline & M1 & $\mathrm{M} 2$ & M3 & M1 & M2 & M3 \\
\hline \multirow[t]{2}{*}{ Bergbau } & -0.043 & -0.023 & -0.023 & 0.070 & 0.062 & 0.061 \\
\hline & $(0.11)$ & $(0.11)$ & $(0.11)$ & $(0.08)$ & $(0.08)$ & $(0.08)$ \\
\hline \multirow[t]{2}{*}{ Verarbeitendes Gewerbe } & $-0.240^{\star \star \star}$ & $-0.195^{* *}$ & $-0.196^{* *}$ & $0.177^{* *}$ & $0.200^{* * *}$ & $0.200^{* * *}$ \\
\hline & $(0.07)$ & $(0.06)$ & $(0.06)$ & $(0.05)$ & $(0.05)$ & $(0.05)$ \\
\hline \multirow[t]{2}{*}{ Energie- und Wasserversorgung } & -0.051 & -0.063 & -0.063 & $0.308^{* * *}$ & $0.277^{\star \star * *}$ & $0.278^{* * *}$ \\
\hline & $(0.08)$ & $(0.08)$ & $(0.08)$ & $(0.05)$ & $(0.05)$ & $(0.05)$ \\
\hline \multirow[t]{2}{*}{ Handel } & -0.130 & -0.095 & -0.095 & $0.322^{* * *}$ & $0.359^{* * *}$ & $0.359^{* * *}$ \\
\hline & $(0.07)$ & $(0.07)$ & $(0.06)$ & $(0.07)$ & $(0.06)$ & $(0.06)$ \\
\hline \multirow[t]{2}{*}{ Kredit- und Versicherungsgewerbe } & 0.110 & -0.009 & -0.007 & $0.376^{* * *}$ & $0.249^{\star * *}$ & $0.248^{* *+}$ \\
\hline & $(0.09)$ & $(0.09)$ & $(0.09)$ & $(0.07)$ & $(0.06)$ & $(0.06)$ \\
\hline \multirow[t]{2}{*}{ Gastgewerbe } & $-0.328^{* \star *}$ & $-0.205^{* *}$ & $-0.207^{* *}$ & -0.113 & 0.008 & 0.010 \\
\hline & $(0.08)$ & $(0.07)$ & $(0.07)$ & $(0.08)$ & $(0.08)$ & $(0.08)$ \\
\hline \multirow[t]{2}{*}{ Verkehr und Lagerei } & -0.132 & -0.127 & -0.127 & 0.080 & 0.076 & 0.077 \\
\hline & $(0.07)$ & $(0.07)$ & $(0.06)$ & $(0.07)$ & $(0.06)$ & $(0.06)$ \\
\hline \multirow{2}{*}{ Grundstück- und Wohnungswesen } & $-0.165^{*}$ & $-0.142^{*}$ & $-0.141^{*}$ & 0.121 & $0.141^{*}$ & $0.140^{*}$ \\
\hline & $(0.07)$ & $(0.06)$ & $(0.06)$ & $(0.06)$ & $(0.06)$ & $(0.06)$ \\
\hline \multirow[t]{2}{*}{ Erziehung und Unterricht } & $-0.215^{*}$ & $-0.233^{* *}$ & $-0.232^{* *}$ & 0.081 & 0.102 & 0.103 \\
\hline & $(0.09)$ & (0.08) & $(0.08)$ & $(0.09)$ & (0.09) & $(0.09)$ \\
\hline \multirow[t]{2}{*}{ Gesundheits- und Sozialwesen } & -0.102 & -0.097 & -0.095 & $0.215^{* *}$ & $0.189^{* *}$ & $0.186^{*}$ \\
\hline & $(0.07)$ & $(0.07)$ & $(0.07)$ & $(0.08)$ & $(0.07)$ & $(0.07)$ \\
\hline \multirow[t]{2}{*}{ Sons. Dienstleistungen } & $-0.186^{*}$ & $-0.170^{*}$ & $-0.169^{*}$ & 0.114 & $0.151^{\star}$ & $0.151^{*}$ \\
\hline & $(0.07)$ & $(0.07)$ & $(0.07)$ & $(0.07)$ & $(0.06)$ & $(0.06)$ \\
\hline \multirow[t]{2}{*}{ Beruflicher Rang } & & $0.597^{\star \star \star *}$ & $0.628^{* * *}$ & & $0.541^{\star \star \star *}$ & $0.525^{\star * *}$ \\
\hline & & $(0.01)$ & $(0.02)$ & & $(0.02)$ & $(0.03)$ \\
\hline \multirow[t]{2}{*}{ Frau * berufl. Rang } & & & $-0.082^{* * t}$ & & & 0.031 \\
\hline & & & $(0.02)$ & & & $(0.03)$ \\
\hline \multirow[t]{2}{*}{ Frau * Bildungsjahre } & & & $-0.009^{* * *}$ & & & -0.004 \\
\hline & & & $(0.00)$ & & & $(0.00)$ \\
\hline \multirow[t]{2}{*}{ Frau * Jahre im Betrieb } & & & 0.000 & & & $0.001^{\star}$ \\
\hline & & & $(0.00)$ & & & $(0.00)$ \\
\hline \multirow[t]{2}{*}{ Frau * Befristung } & & & $0.031^{* \star}$ & & & $0.073^{* * *}$ \\
\hline & & & $(0.01)$ & & & $(0.02)$ \\
\hline \multirow[t]{2}{*}{ Konstante } & $0.382^{* *}$ & $0.738^{\star \star \star}$ & $0.691^{* * *}$ & $0.274^{*}$ & $0.610^{* \star *}$ & $0.599^{* * *}$ \\
\hline & $(0.12)$ & $(0.11)$ & $(0.11)$ & $(0.13)$ & $(0.11)$ & $(0.11)$ \\
\hline \multirow[t]{2}{*}{ sd(Frau) } & $0.111^{\star \star \star}$ & $0.110^{* \star *}$ & $0.110^{* * *}$ & $0.094^{* * *}$ & $0.089^{\star \star \star *}$ & $0.089^{* * *}$ \\
\hline & $(0.00)$ & $(0.00)$ & $(0.00)$ & $(0.00)$ & $(0.00)$ & $(0.00)$ \\
\hline \multirow[t]{2}{*}{ sd(Konstante) } & $0.216^{\star \star *}$ & $0.201^{* * *}$ & $0.200^{* * *}$ & $0.185^{* \star *}$ & $0.160^{\star \star *}$ & $0.160^{* * *}$ \\
\hline & $(0.00)$ & $(0.02)$ & $(0.00)$ & $(0.00)$ & $(0.00)$ & $(0.00)$ \\
\hline \multirow[t]{2}{*}{ Korrelation(Frau, Konstante) } & $-0.247^{\star \star *}$ & $-0.205^{\star \star \star}$ & $-0.201^{* \star \star}$ & $-0.364^{* * *}$ & $-0.312^{\star \star *}$ & $-0.321^{\star \star \star *}$ \\
\hline & $(0.02)$ & $(0.02)$ & $(0.02)$ & $(0.00)$ & $(0.04)$ & $(0.04)$ \\
\hline \multirow[t]{2}{*}{ sd(Residuen) } & $0.259^{\star \star \star *}$ & $0.236^{\star \star \star}$ & $0.236^{* * *}$ & $0.228^{* * *}$ & $0.208^{\star \star *}$ & $0.208^{* * *}$ \\
\hline & $(0.00)$ & $(0.00)$ & $(0.00)$ & $(0.00)$ & $(0.01)$ & $(0.01)$ \\
\hline $\bar{N}$ & 98.401 & 98.401 & 98.401 & 40.410 & 40.410 & 40.410 \\
\hline
\end{tabular}


Abbildung A1: Effekt der Interaktion von Frauen mit dem Frauenanteil im Management, Ostdeutschland

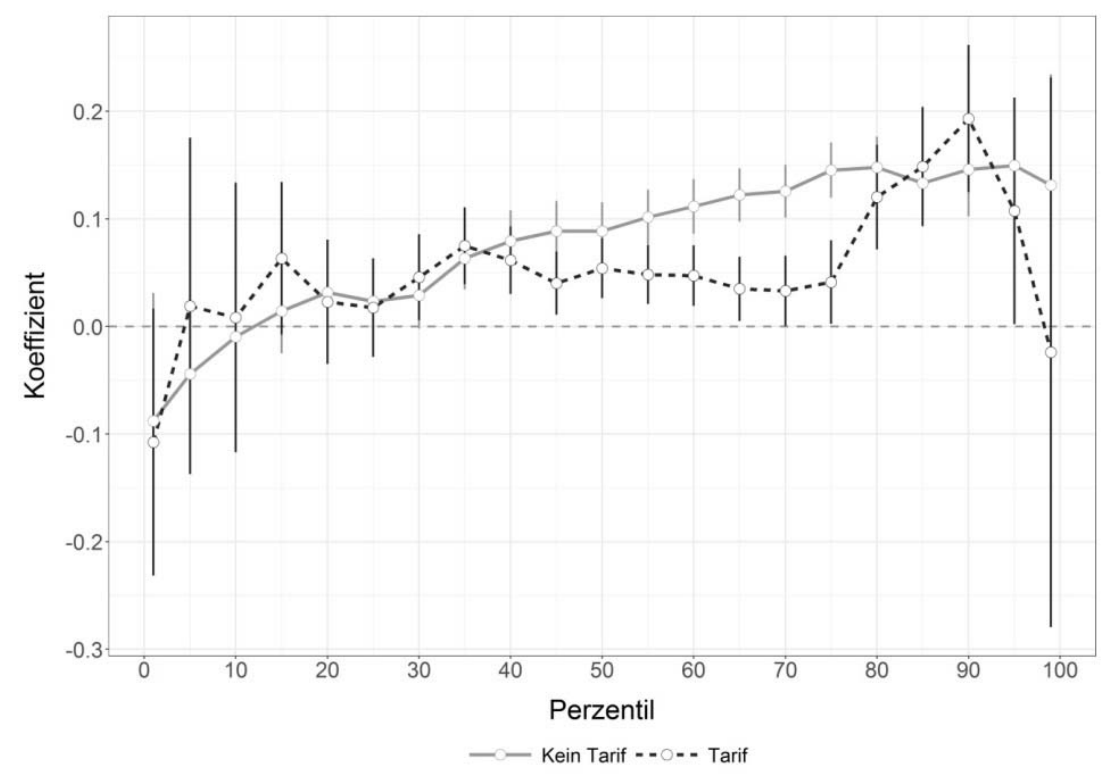

Abbildung A2: Effekt der Interaktion von Frauen mit der Differenz im Anteil an Hochschulabschlüssen, Ostdeutschland

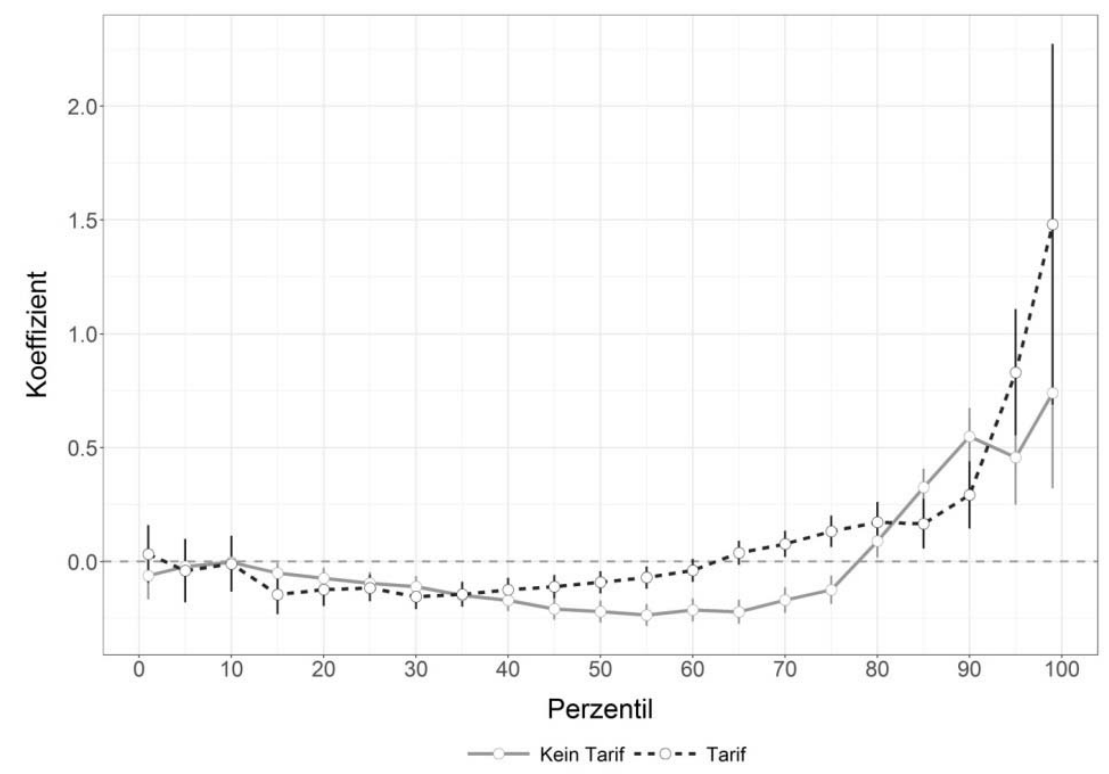


DuEPublico

Duisburg-Essen Publications online
UNIVERSITÄT

$D_{E} U_{S} I_{S} B_{N} U$ R G

offen im Denken

$\mathbf{u b} \mid$\begin{tabular}{c|c} 
universitäts \\
bibliothek
\end{tabular}

Dieser Text wird über DuEPublico, dem Dokumenten- und Publikationsserver der

Universität Duisburg-Essen, zur Verfügung gestellt. Die hier veröffentlichte Version der EPublikation kann von einer eventuell ebenfalls veröffentlichten Verlagsversion abweichen.

DOI: $\quad$ 10.3224/indbez.v24i3.04

URN: urn:nbn:de:hbz:464-20200703-100317-0

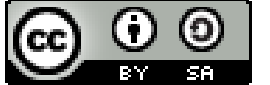

Dieses Werk kann unter einer Creative Commons Namensnennung

- Weitergabe unter gleichen Bedingungen 4.0 Lizenz (CC BY-SA 4.0) genutzt werden. 